\title{
Combined Core Pillar Concept for Earthquake Resistant Building
}

\author{
Shubham Gupta \\ Pursuing civil engineering from Galgotia's College Of Engineering \& Technology, greater noida(U.P) 201306- \\ India
}

\begin{abstract}
The main aim of this paper is to introduce "Combined Core Pillar Concept" for earthquake resistant design of a building. A 12 storey building model of height $43 \mathrm{~m}$ is taken in which this concept is applied.All other methods which has been used in this model are-building with friction isolator (FI), with rubber bearing $(R B)$, with shearwall (fixed base), with shearwall having base as rubber bearing and friction isolator, with cross bracing \& with k-type bracing. A comparative study of the model with these different techniques is done with the help of software SAP 2000.The method used for the analysis is RESPONSE SPECTRUM METHOD. Here the design spectra recommended by Indian Standard Code IS 1893-2002(PART I) is used. From comparative study the Combined Core Pillar concept is found to be most effective.
\end{abstract}

\section{Introduction}

The main challenge in earthquake resistant design is to reduce the earthquake forces so that an economic \& safe design of members of the structure can be done.Basically two criterias must be fulfilled the strength criteria \& the deflection criteria.To resist the earthquake forces many methods have been used in buildings like use of shearwalls at appropriate positions in the buildings, use of bracings (cross bracing, $\mathrm{k}$ type bracing etc). In all these cases the value of base shear, base moments are high \& according to these values the different components of building are designed, obviously the sectional requirement of the components in these cases are high to resist such high forces. Since in seismic analysis of a building base shear is distributed to the different floors according to the floor heights \& then these floor forces are distributed among the lateral force resisting elements at that floor so if the base shear is high then the sectional requirement of these components will also high.So to reduce the base shear \& the inertia forces induced in the structure due to earthquake, base isolation technique is frequently used in practice. In base isolation technique the base of the structure is isolated so that the fundamental period of the structure is shifted out of the dangerous resonance range \& concentration of the deformation demand at the isolation system.

But I have used a different method to shift the fundamental period of the structure that is "COMBINED CORE PILLAR CONCEPT". So to compare response of building with different techniques used, a parametric study on reinforced concrete (RC) building is done. For this purpose the different techniques used in a same model are :

1. RC building model with fixed column base.

2. RC building model with Rubber bearing (RB)column base .

3. RC building model with friction isolated(FI) column base.

4. RC building model with shearwalls at corners having fixed base.

5. RC building model with shearwalls at corners with rubber bearings base.

6. RC building model with shearwalls at corners with friction isolator as base.

7. RC building model with cross bracings.

8. RC building model with k-type bracings.

9. RC building model with combined core pillar concept having hinged base of core steel column.

10. RC building model with combined core pillar concept having fixed base of core steel column. 
Design Spectra:

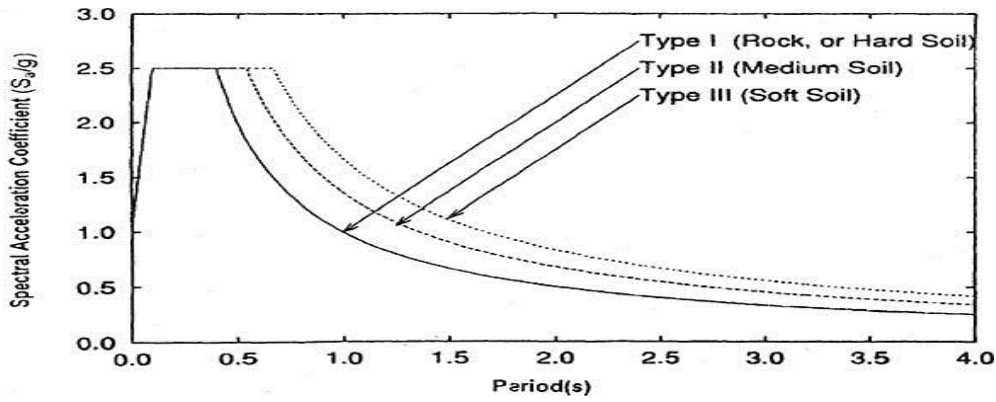

This is the DESIGN SPECTRA recommended by IS 1893-PART(I).From this, type II(medium soil) is selected for the analysis purpose.On the basis of the fundamental time period of the structure the value of $\left(\mathrm{S}_{\mathrm{a}} / \mathrm{g}\right)$ can be selected from this curve according to the soil type selected for the analysis. Here the empirical relation is also presented recommended by the code.

For rocky, or hard soil sites

$$
\frac{S_{\mathrm{a}}}{g}= \begin{cases}1+15 T, & 0.00 \leq T \leq 0.10 \\ 2.50 & 0.10 \leq T \leq 0.40 \\ 1.00 / T & 0.40 \leq T \leq 4.00\end{cases}
$$

For medium soil sites

$$
\frac{S_{\mathrm{a}}}{g}= \begin{cases}1+15 T ; & 0.00 \leq T \leq 0.10 \\ 2.50 & 0.10 \leq T \leq 0.55 \\ 1.36 / T & 0.55 \leq T \leq 4.00\end{cases}
$$

For soft soil sites

$$
\frac{S_{\mathrm{s}}}{g}= \begin{cases}1+15 T, & 0.00 \leq T \leq 0.10 \\ 2.50 & 0.10 \leq T \leq 0.67 \\ 1.67 / T & 0.67 \leq T \leq 4.00\end{cases}
$$

ZONE FACTOR:

Table 2 Zome Factor, $Z$

(Clause 6.4.2)

\begin{tabular}{ccccc}
\hline $\begin{array}{c}\text { Seismic } \\
\text { Zone }\end{array}$ & I & III & IV & V \\
$\begin{array}{c}\text { Seismic } \\
\text { Intensity }\end{array}$ & Low & Moderate & Severe & $\begin{array}{c}\text { Very } \\
\text { Severe } \\
Z\end{array}$ \\
\hline
\end{tabular}

From this seismic zone $\mathrm{v}$ is selected for the analysis of building model using RESPONSE SPECTRUM METHOD.

DAMPING: The design spectra is for 5\% damping which has been used in the analysis.

\section{Modeling Of Building And Result Analysis:}

To evaluate the seismic response of the building, elastic analyses were performed by the response spectrum method using the computer program SAP2000. The seismic analyses of the building are carried out separately in the longitudinal and transverse directions. However seismic responses only for $\mathrm{x}-$ direction are comparatively presented in this paper for the sake of brevity. Floor plan of 12 storey building is $12 \times 18 \mathrm{~m}$.Degree of freedom at the base nodes are fixed for fixed base case and for base isolation, the friction isolators \& rubber isolator is used.The parameters selected to define the utilized rubber \& friction isolators in SAP2000 program are as follows: 
Non-linear link type: Rubber Bearing->

1. U1 linear effective stiffness $=1500000 \mathrm{KN} / \mathrm{m}$

2. U2 \& U3 linear effective stiffness: $800 \mathrm{KN} / \mathrm{m}$

3. U2 \& U3 nonlinear stiffness : $2500 \mathrm{KN} / \mathrm{m}$

4. U2 \& U3 yield strength : $80 \mathrm{KN}$

5. U2 \& U3 post yield stiffness ratio: 0.1

Non-linear link type friction isolator->

1. U1 linear effective stiffness : $15000000 \mathrm{KN} / \mathrm{m}$.

2. U2 \& U3 non linear stiffness: $15000 \mathrm{KN} / \mathrm{m}$

3. U2 \& U3 friction coefficient, slow: 0.03 , fast:0.05

4. Rate parameter: 40

5. U2 \& U3 radius of sliding surface: 2.23

Columns and beams are modeled with frame elements, slabs and structural walls are modeled with shell elements. Slab has been considered as a rigid diaphragm in each storey level. In the analysis Young's modulus and the unit weight of concrete are taken to be $28000 \mathrm{MPa}$ and $25 \mathrm{KN} / \mathrm{m}^{3}$ respectively. The damping ratio is assumed as $5 \%$ in all modes. The reference peak ground acceleration is taken to be $.4 \mathrm{~g}$ that is recommended in IS code. Thus it is assumed that the building is suited in high seismicity zone. Seismic analysis of the building accounting for the influence of the local ground conditions is carried out with the help of the design spectra of IS code.

Figures of model with different techniques are shown below:

1. $\mathrm{RC}$ building model with combined core pillar concept having hinged base of core steel column $=$ model 1

2. RC building model with combined core pillar concept having fixed base of core steel column $=$ model 2

3. $\mathrm{RC}$ building model with fixed column base $=$ model 3

4. $\mathrm{RC}$ building model with Rubber bearing $(\mathrm{RB})$ column base $=$ model 4

5. $\mathrm{RC}$ building model with friction isolated(FI) column base $=$ model 5

6. $\mathrm{RC}$ building model with shearwalls at corners having fixed base $=$ model 6

7. $\mathrm{RC}$ building model with shearwalls at corners with rubber bearings at base $=\operatorname{model} 7$

8. $\mathrm{RC}$ building model with shearwalls at corners with friction isolator at base $=$ model 8

9. $\mathrm{RC}$ building model with cross bracings $=$ model 9

10. $\mathrm{RC}$ building model with $\mathrm{k}$-type bracings $=$ model 10

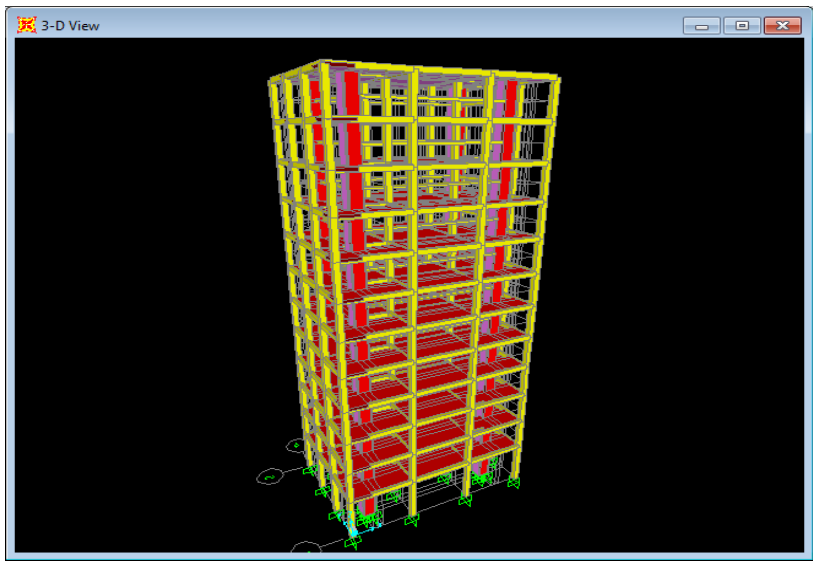

MODEL 1(a)

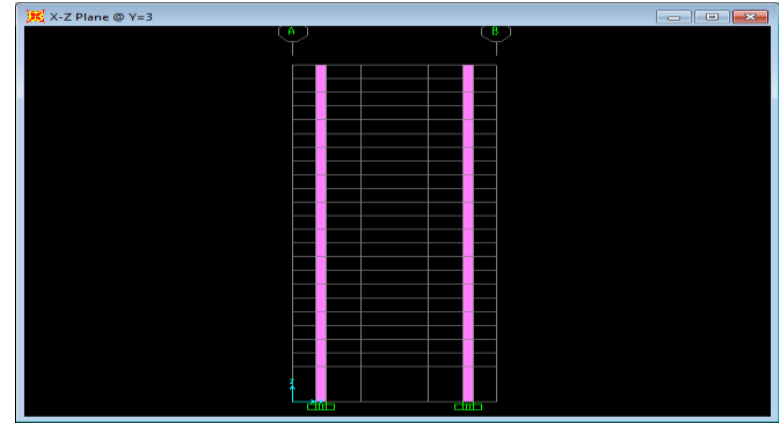

MODEL 1(b) 


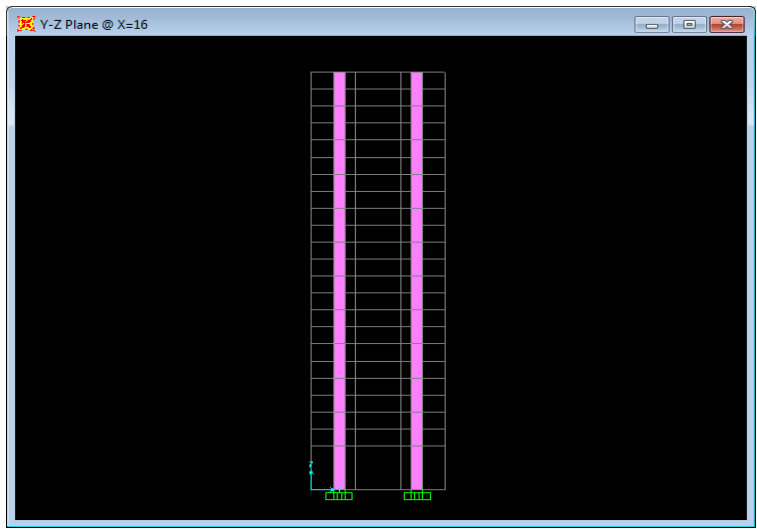

MODEL 1(c)
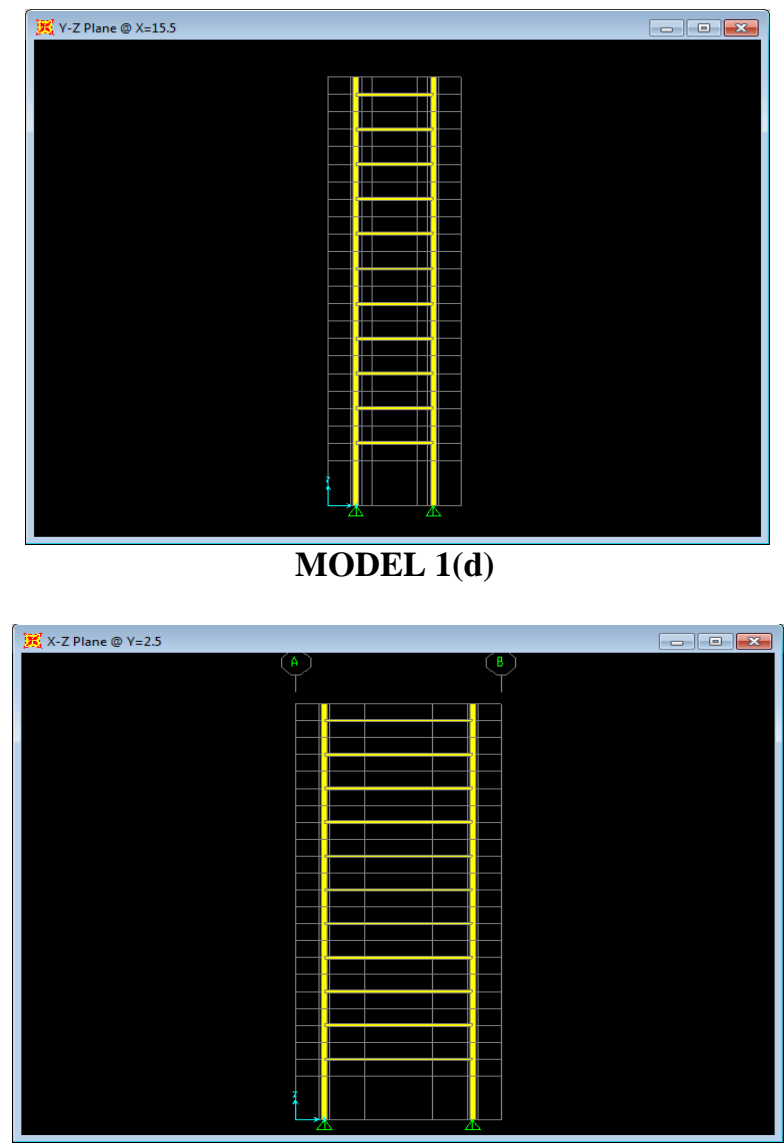

MODEL 1(e)

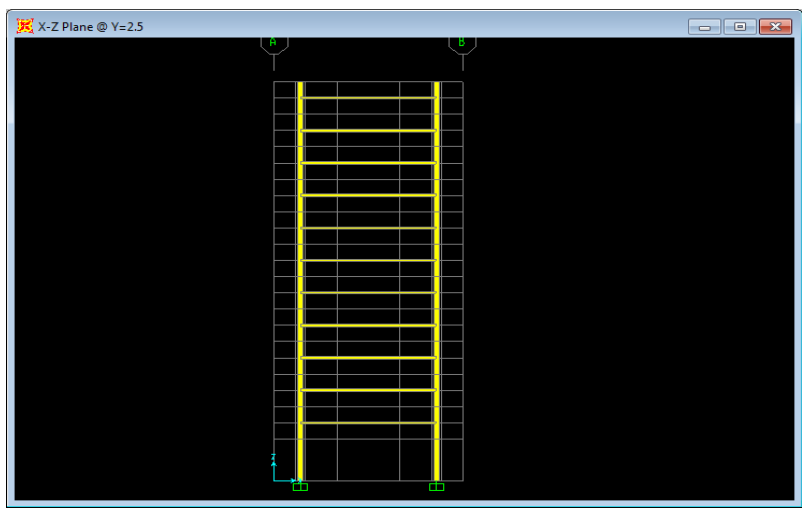

MODEL 2 


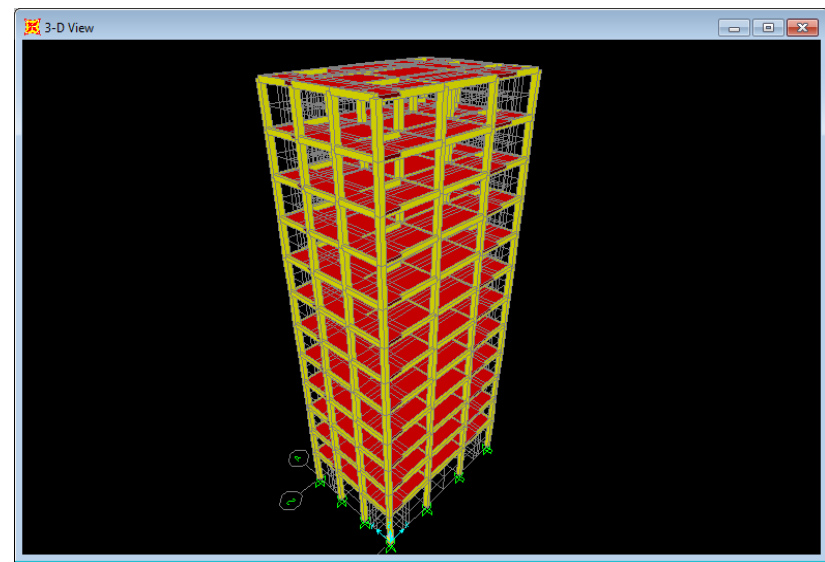

MODEL 3(a)

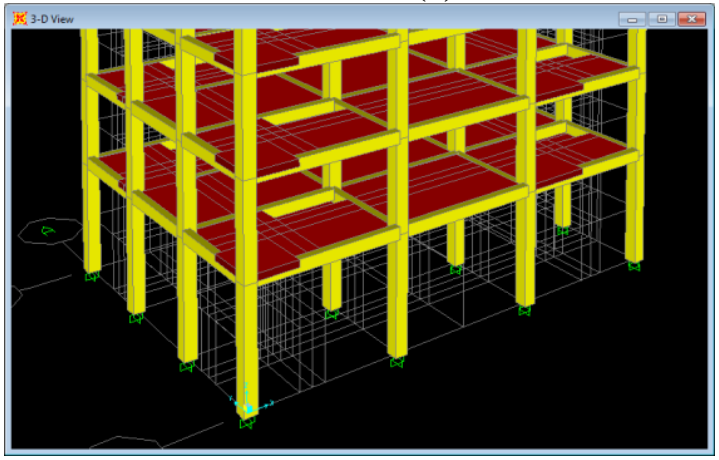

MODEL 3(b)

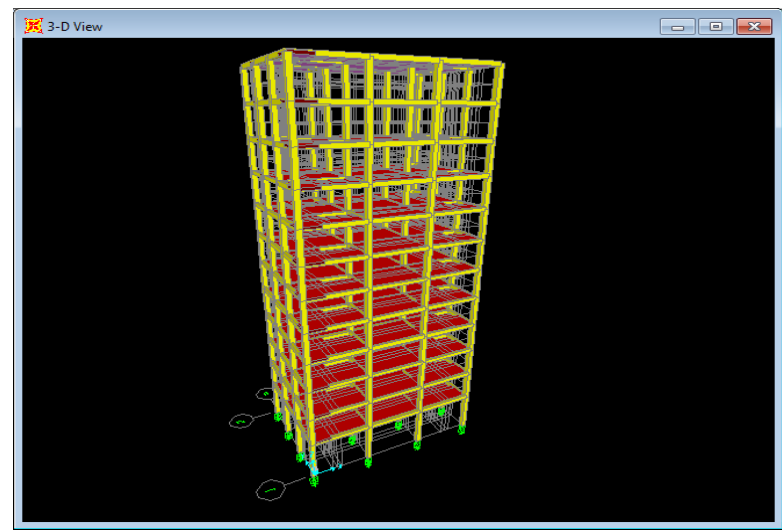

MODEL 4(a)

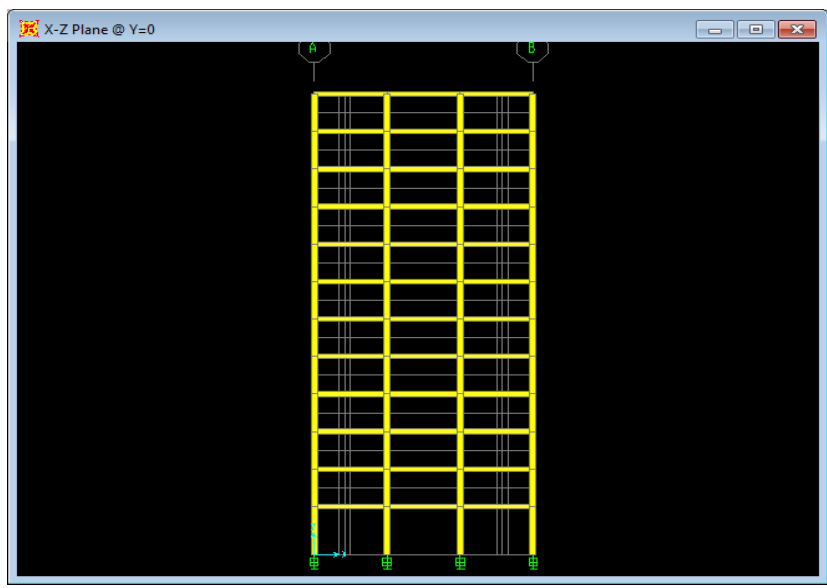

MODEL 4(b) 


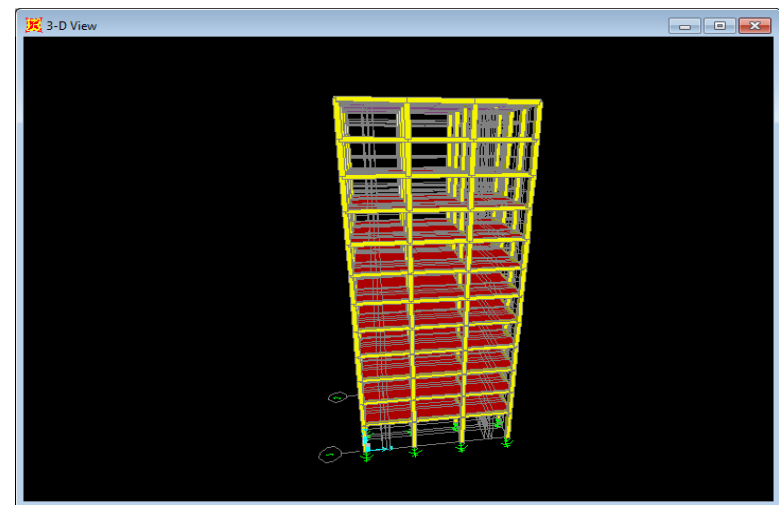

MODEL 5(a)

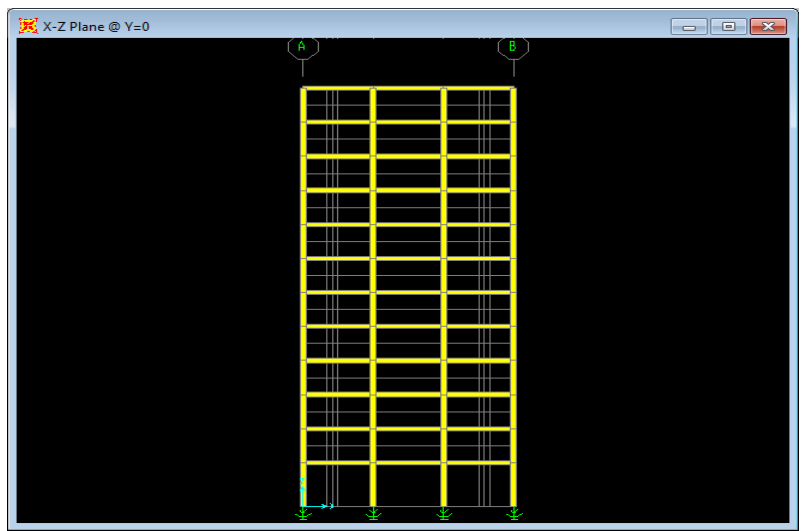

MODEL 5(b)

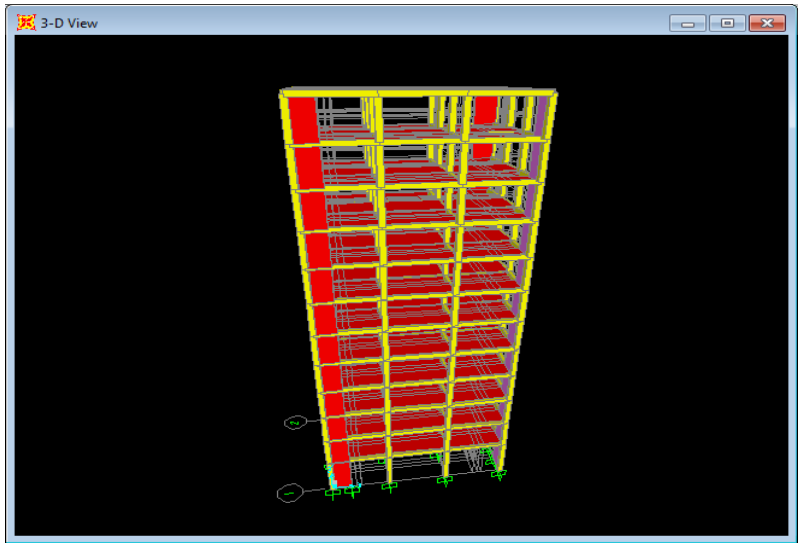

MODEL 6(a)

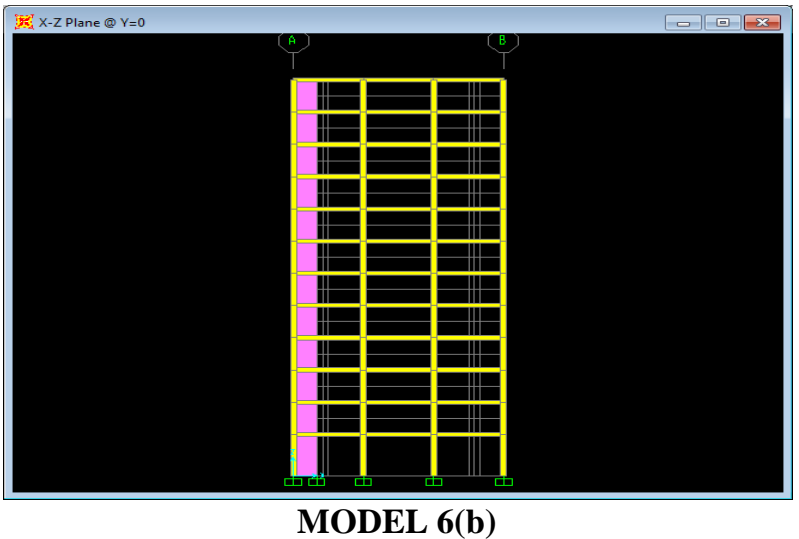




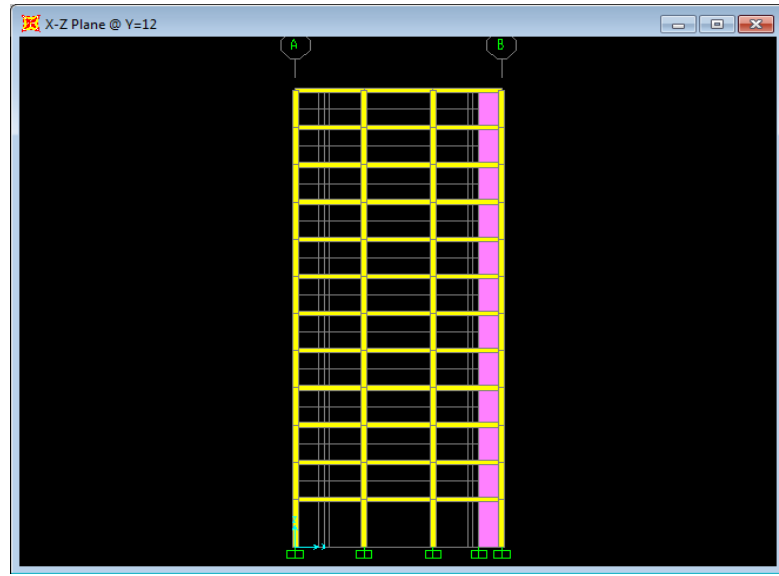

MODEL 6(c)
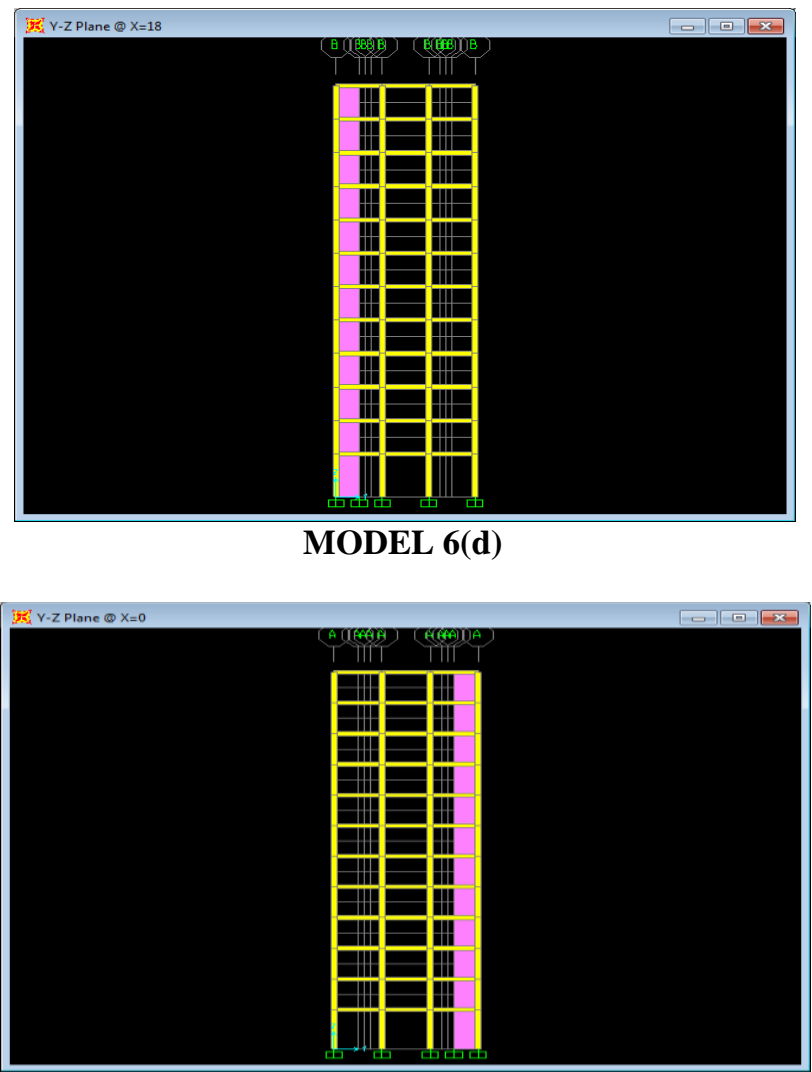

MODEL 6(e)

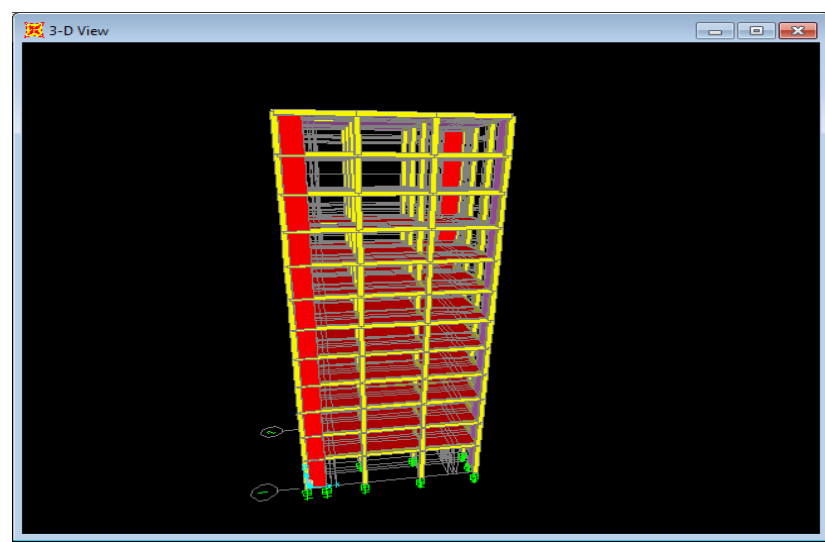

MODEL 7(a) 


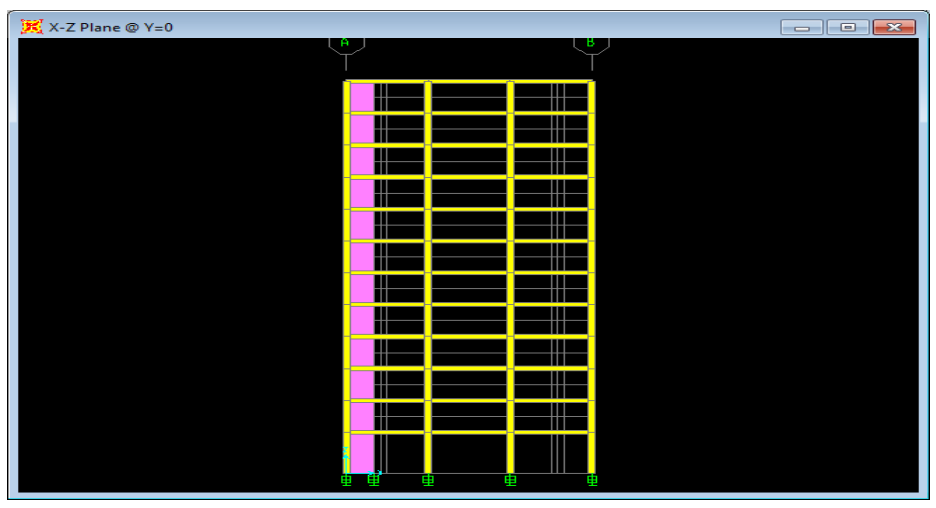

MODEL 7(b)

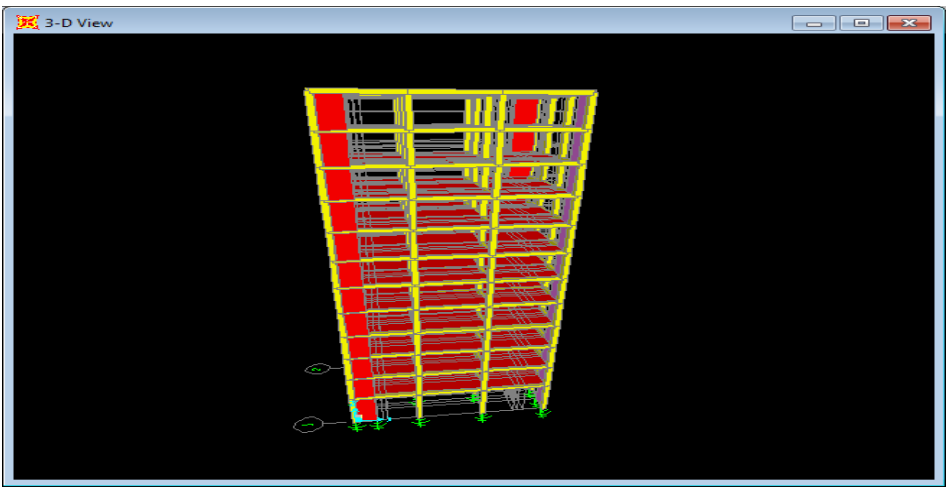

MODEL 8(a)

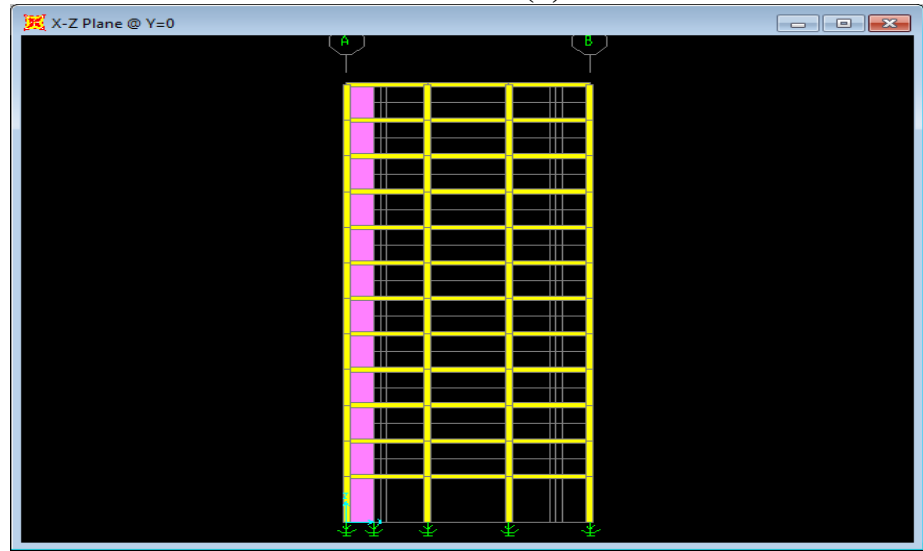

MODEL 8(b)

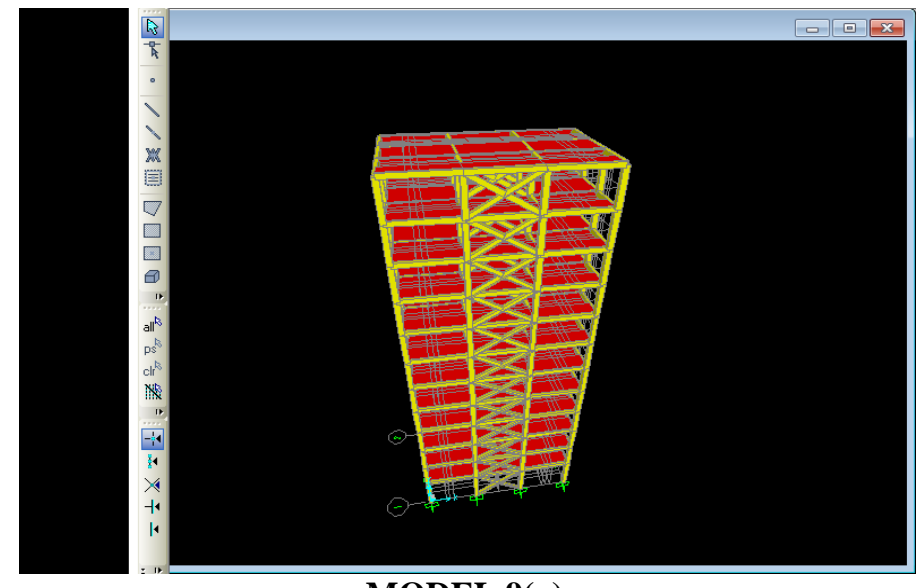

MODEL 9(a) 


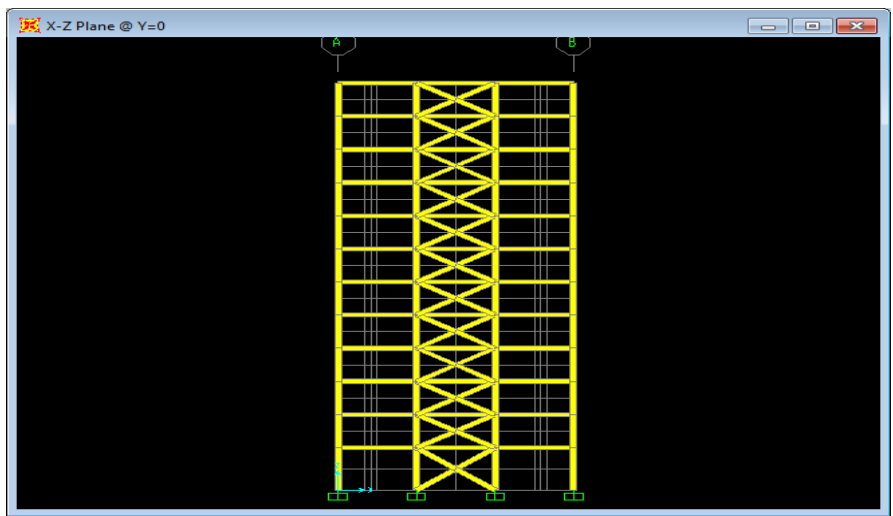

MODEL 9(b)

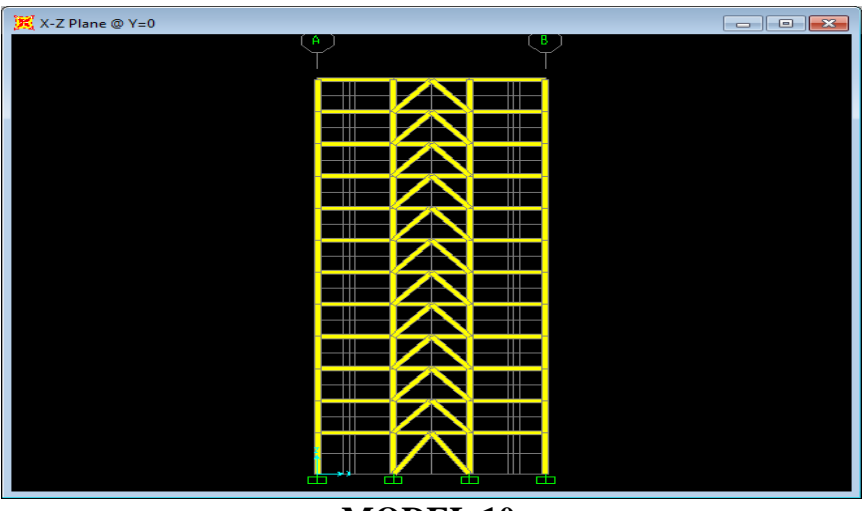

Results Of Model 3:

MODEL 10

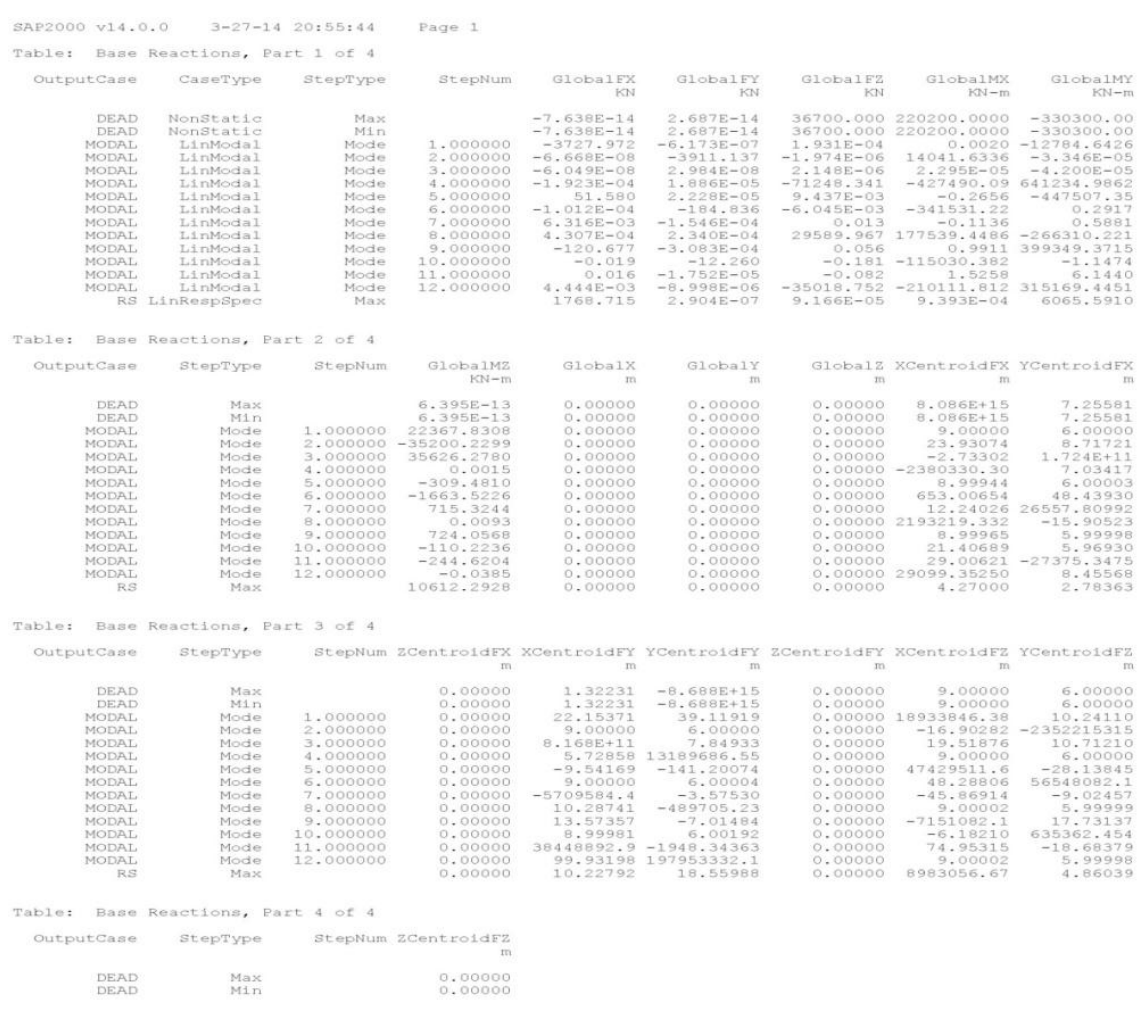




$\begin{array}{lrrr}\text { SAP2000 v14.0.0 } & 3-27-14 & 20: 55: 45 & \text { Page } 2 \\ \text { Table: Base Reactions, Part } 4 \text { of 4, Cont. } \\ \text { OutputCase } & \text { StepType } & \text { StepNum ZCentroidEz } \\ & & & \\ \text { MODAL } & \text { Mode } & 1.000000 & 0.00000 \\ \text { MODAL } & \text { Mode } & 2.000000 & 0.00000 \\ \text { MODAL } & \text { Mode } & 3.000000 & 0.00000 \\ \text { MODAL } & \text { Mode } & 4.000000 & 0.00000 \\ \text { MODAL } & \text { Mode } & 5.000000 & 0.00000 \\ \text { MODAL } & \text { Mode } & 6.000000 & 0.00000 \\ \text { MODAL } & \text { Mode } & 7.000000 & 0.00000 \\ \text { MODAL } & \text { Mode } & 8.000000 & 0.00000 \\ \text { MODAL } & \text { Mode } & 9.000000 & 0.00000 \\ \text { MODAL } & \text { Mode } & 10.000000 & 0.00000 \\ \text { MODAL } & \text { Mode } & 11.000000 & 0.00000 \\ \text { MODAL } & \text { Mode } & 12.000000 & 0.00000 \\ \text { RS } & \text { Max } & & 0.00000\end{array}$

SAP2000 v14.0.0 3-27-14 21:04:58 Page 1

Table: Modal Periods and Frewencles

\begin{tabular}{|c|c|c|c|c|c|c|}
\hline OutputCase & Steplype & StepNun & $\begin{array}{r}\text { Peried } \\
\text { Sec }\end{array}$ & $\begin{array}{r}\text { Erequency } \\
\text { Cyc/sec }\end{array}$ & $\begin{array}{l}\text { CircEreq } \\
\mathrm{rad} / \mathrm{sec}\end{array}$ & $\begin{array}{l}\text { Eigenvalue } \\
\text { rad2/sec? }\end{array}$ \\
\hline MODAL & Mode & 1,000000 & 0.732199 & $1.365 \mathrm{TE}+00$ & $8.5813 \mathrm{E}+00$ & $7,3636 \mathrm{E}+01$ \\
\hline MODRL & Mode & 2.000000 & 0.714854 & $1.3989 \mathrm{E}+00$ & $8.7895 \mathrm{E}+00$ & $7.7255 \mathrm{E}+01$ \\
\hline MODAL & Mode & 3.000000 & 0.624660 & $1.600 \mathrm{BE}+00$ & $1.0058 \mathrm{E}+01$ & $1.0117 \mathrm{TE}+02$ \\
\hline MODAL & Mode & 4.000000 & 0.159090 & $6.2850 \mathrm{E}+00$ & $3.94958+01$ & \\
\hline MODAL & Mode & 5,000000 & 0.143440 & $6.9715 \mathrm{E}+00$ & $4.3803 \mathrm{E}+01$ & $1.9187 \mathrm{E}+0$ \\
\hline MOLRL & Mode & 6.000000 & 0.125793 & $7.9496 \mathrm{E}+00$ & $4.9949 \mathrm{E}+01$ & $2.4949 \mathrm{E}+0$ \\
\hline MODAL & Mode & 7,000000 & 0.117894 & $8.4822 \mathrm{~F}+00$ & $5.32958+01$ & $2.8404 \mathrm{E}+\mathrm{C}$ \\
\hline MODAL & Mode & 8.000000 & 0.101793 & $9.8239 \mathrm{E}+00$ & $6.17258+01$ & $3.8100 \mathrm{E}+$ \\
\hline MODAL & Mode & 9.000000 & 0.091918 & $1,0879 \mathrm{E}+01$ & $6.8357 E+01$ & $4.6726 \mathrm{E}+0$ \\
\hline MODM: & Mode & 10.000000 & 0.085886 & $1.1643 \mathrm{E}+01$ & $7.3157 \mathrm{E}+01$ & $5.3520 \mathrm{E}+0$ \\
\hline & Mode & 11,000000 & 0.079541 & $1.2572 \mathrm{E}+01$ & $7,89938+01$ & $6.2398 \mathrm{E}+\mathrm{C}$ \\
\hline & Mode & 12.000000 & 0.079491 & $1.2580 \mathrm{E}+01$ & $7.9042 E+01$ & $6.2479 E+03$ \\
\hline
\end{tabular}

BASE REACTIONS

\begin{tabular}{|ll|l|l|}
\hline \multicolumn{5}{|c|}{ RS Lin Resp. Spec.(max) } \\
\hline GLOBAL & FX (KN) & GLOBAL MY (KN-m) & GLOBAL MZ (KN-m) \\
\hline 1768.715 & & $6065.591 \quad 10612.2928$ \\
\hline
\end{tabular}




\section{Results Of Model 4:}
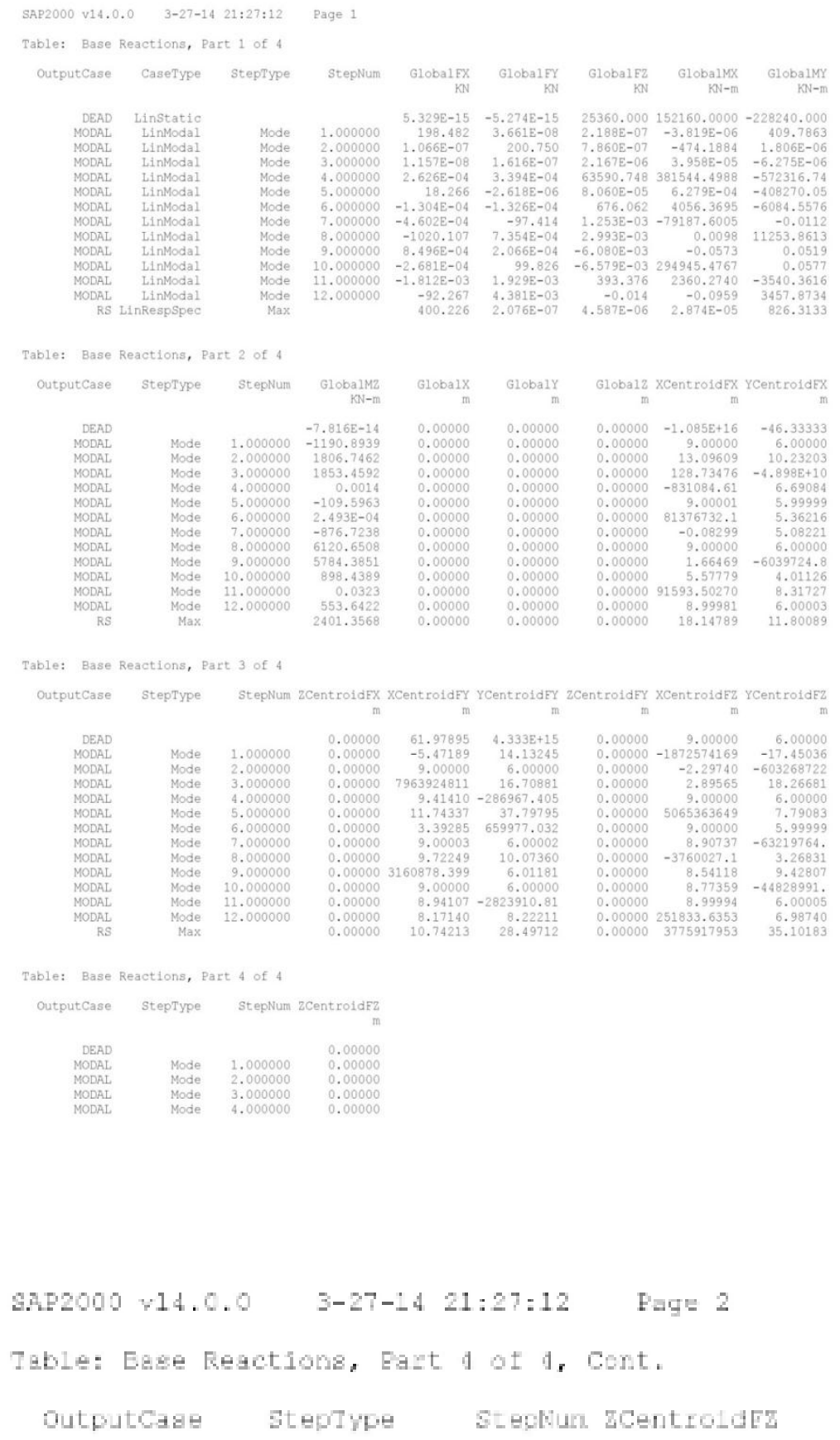


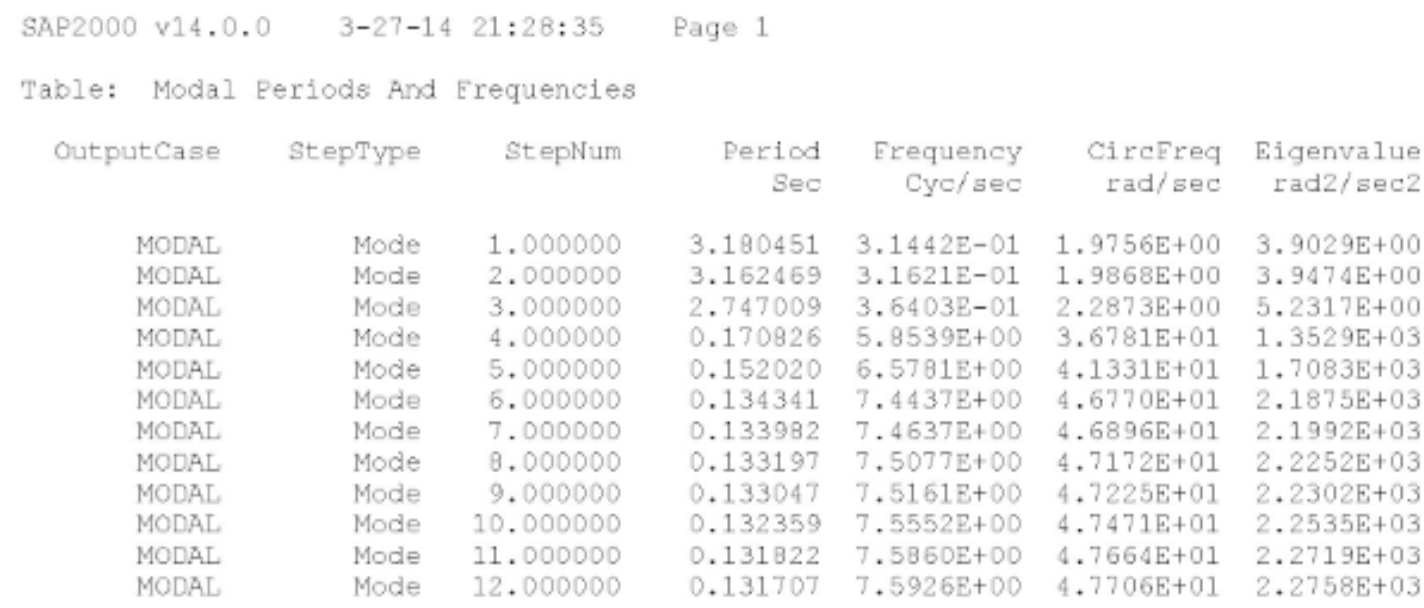

\begin{tabular}{|l|l|l|}
\hline \multicolumn{4}{|c|}{ BASE REACTIONS } \\
\hline \multicolumn{3}{|c|}{ RS Lin Resp. spec.(max) } \\
\hline GLOBAL FX (KN) & GLOBAL MY (KN-m) & GLOBAL MZ(KN-m) \\
\hline 400.226 & 826.313 & 2401.356 \\
\hline
\end{tabular}

\section{Results Of Model 5:}

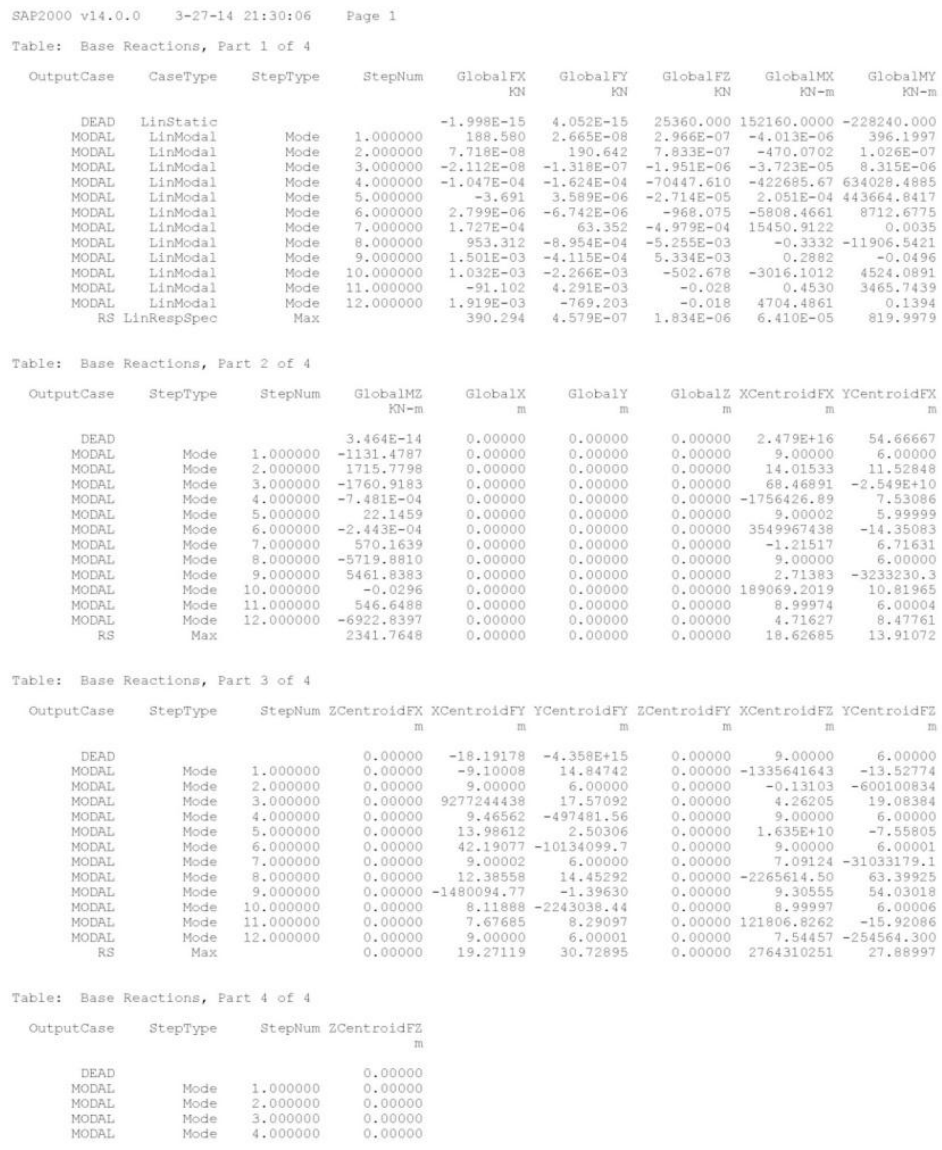




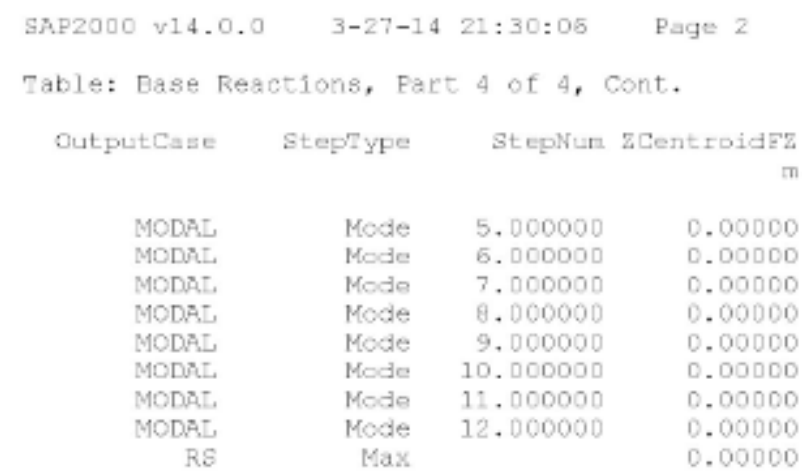

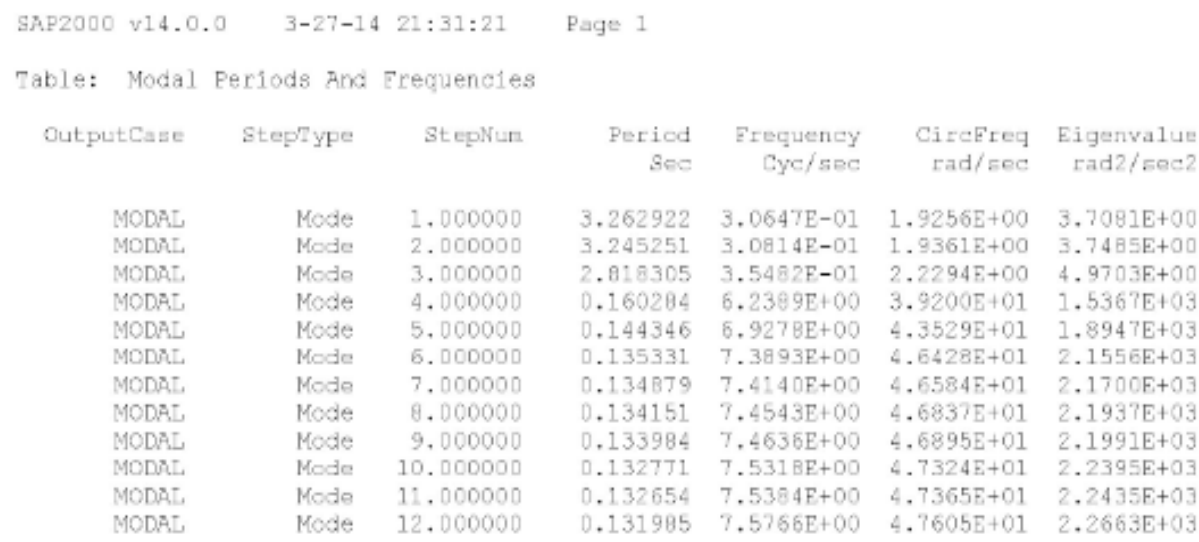

BASE REACTIONS

\begin{tabular}{|c|c|c|}
\hline \multicolumn{3}{|c|}{ RS Lin Resp. Spec. (max) } \\
\hline GLOABAL FX $(\mathrm{KN})$ & GLOBAL MY (KN-m) & GLOBAL $\quad$ MZ (KN-m) \\
\hline 390.294 & 820 & 2341.7648 \\
\hline
\end{tabular}

\section{Results Of Model 6:}




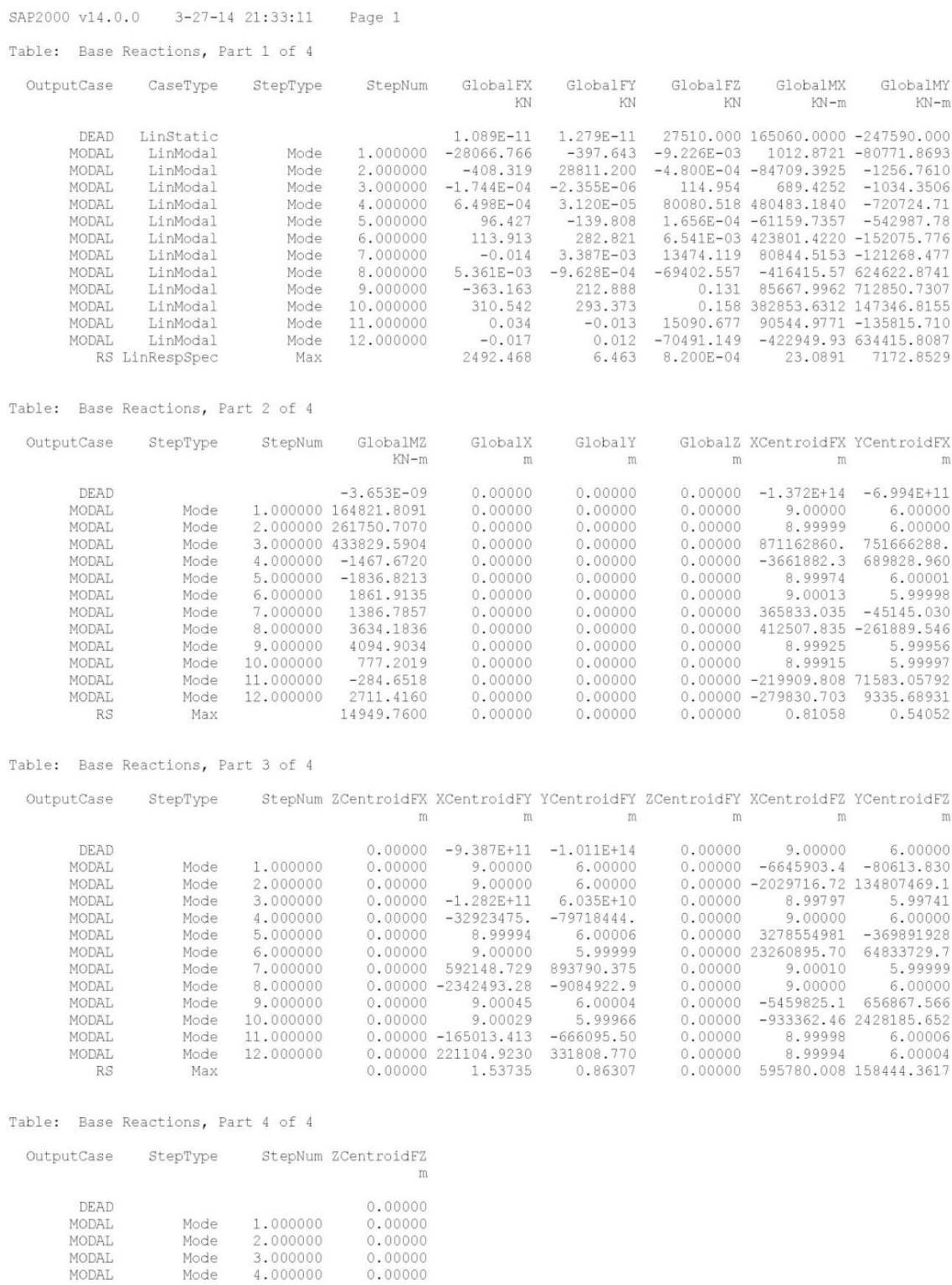




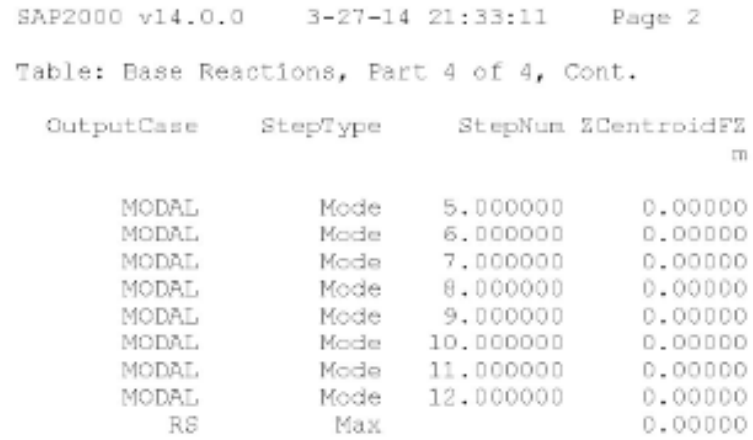

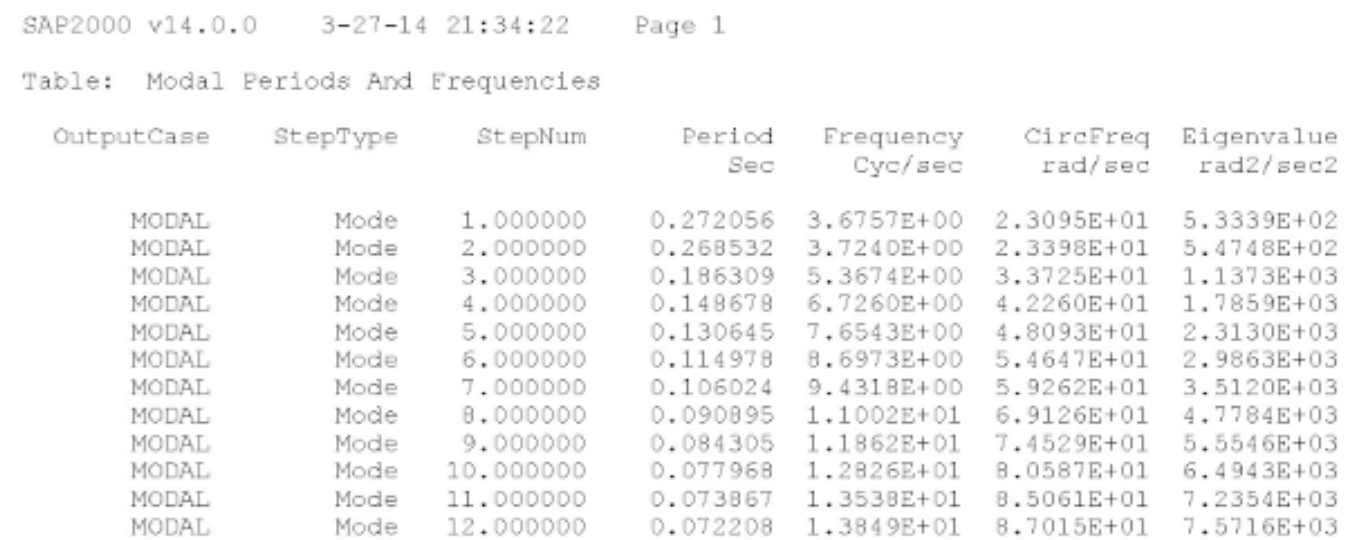

BASE REACTIONS

\begin{tabular}{|c|c|c|}
\hline \multicolumn{3}{|c|}{ BASE REACTIONS } \\
\hline \multicolumn{3}{|c|}{ RS Lin Resp. spec. (max) } \\
\hline GLOBAL FX $(\mathrm{KN})$ & GLOBAL MY (KN-m) & GLOBAL MZ (KN-m) \\
\hline 2492.468 & 7172.85 & 14949.76 \\
\hline
\end{tabular}

RESULTS OF MODEL 7: 
SAP2000 v14.0.0 3-27-14 21:37:09

Table: Base Reactions, Part 1 of 4

Outputcase CaseType StepType

$\begin{array}{rr}\text { DEAD } & \text { LinStatic } \\ \text { MODAL } & \text { LinModal }\end{array}$

$\begin{array}{lll}\text { MODAL } & \text { LinModal } & \text { Mode } \\ \text { MODAL } & \text { LinModal } & \text { Mode }\end{array}$

MODAL LinModal Mode

MODAL LinModal Mode

MODAL LinModal $\quad$ Mode

MODAL LinModal Mode

$\begin{array}{lll}\text { MODAL } & \text { LinModal } & \text { Mode } \\ \text { MODAL } & \text { LinModal } & \text { Mode }\end{array}$

MODAL LinModal Mode

MODAL LinModal $\quad$ Mode

RS LinRespspec Max

Page 1

$\begin{array}{rrrrrr}\text { StepNum GlobalFX } & \text { GlobalFY } & \text { GlobalFZ } & \text { GlobalMX } & \text { GlobalMY } \\ & \mathrm{KN} & \mathrm{KN} & \mathrm{KN} & \mathrm{KN}-\mathrm{m} & \mathrm{KN}-\mathrm{m}\end{array}$

$\begin{array}{rrrrrr}1.000000 & -3.879 \mathrm{E}-11 & -1.166 \mathrm{E}-10 & 27510.000 & 165060.0000 & -247590.000 \\ -247.129 & -4.289 & -1.597 \mathrm{E}-06 & -2.1685 & -506.6604\end{array}$

$\begin{array}{llllll}2.000000 & -4.331 & 249.502 & 2.339 \mathrm{E}-06 & -580.7239 & -5.2788\end{array}$

$\begin{array}{rrrrrr}3.000000 & 4.676 \mathrm{E}-08 & -2.378 \mathrm{E}-07 & 0.069 & 0.4164 & -0.6246\end{array}$

$\begin{array}{llllll}4.000000 & 1.514 \mathrm{E}-05 & 2.122 \mathrm{E}-06 & -1225.038 & -7350.2273 & 11025.3405\end{array}$

$\begin{array}{llllll}5.000000 & -1041.728 & 8.025 & -6.150 \mathrm{E}-05 & -2145.1826 & -390.2015\end{array}$

$\begin{array}{llllll}5.000000 & -15.285 & -1026.399 & -3.022 \mathrm{E}-05 & -3777.8829 & -3286.2448\end{array}$

$\begin{array}{llllll}7.000000 & -1.568 \mathrm{E}-05 & -2.116 \mathrm{E}-05 & -914.969 & -5489.8307 & 8234.7307\end{array}$

$\begin{array}{llllll}8.000000 & -4.041 \mathrm{E}-03 & -3.935 \mathrm{E}-03 & -72520.413 & -435121.91 & 652683.4805\end{array}$

$\begin{array}{llllll}9.000000 & -46.767 & 14.286 & -8.354 \mathrm{E}-06 & 48582.1169 & 507486.1620\end{array}$

$\begin{array}{llllll}10.000000 & -1.541 \mathrm{E}-06 & 1.170 \mathrm{E}-04 & 572.832 & 3436.9843 & -5155.4831\end{array}$

$\begin{array}{rrrrrr}11.000000 & -563.612 & 20.368 & -0.019 & 6711.4344 & 38029.1123 \\ 12.000000 & -388.298 & 7.370 & -0.031 & 8458.5093 & 2297.8560\end{array}$

$\begin{array}{rrrrr}474.460 & 0.558 & 6.605 \mathrm{E}-05 & 23.4981 & 972.6432\end{array}$

Table: Base Reactions, Part 2 of 4

\begin{tabular}{|c|c|c|c|c|c|c|c|c|}
\hline OutputCase & StepType & StepNum & $\begin{array}{r}\text { GlobalMz } \\
\mathrm{KN}-\mathrm{m}\end{array}$ & $\begin{array}{r}\text { GlobalX } \\
\mathrm{m}\end{array}$ & $\begin{array}{r}\text { Globaly } \\
\mathrm{m}\end{array}$ & $\begin{array}{r}\text { Globalz } \\
\mathrm{m}\end{array}$ & $\begin{array}{r}\text { XCentroidEX } \\
\mathrm{m}\end{array}$ & $\begin{array}{r}\text { YCentroidEX } \\
\mathrm{m}\end{array}$ \\
\hline DEAD & & & $-1.479 \mathrm{E}-07$ & 0.00000 & 0.00000 & 0.00000 & $1.238 \mathrm{E}+12$ & $-1.386 \mathrm{E}+11$ \\
\hline MODAL & Mode & 1.000000 & 1444.1694 & 0.00000 & 0.00000 & 0.00000 & 9.00000 & 6.000 \\
\hline MODAL & Mode & 2.000000 & 2271.5050 & 0.00000 & 0.00000 & 0.00000 & 9.00000 & 6.00000 \\
\hline MODAL & Mode & 3.000000 & 2471.8447 & 0.00000 & 0.00000 & 0.00000 & -2768232866 & $-1.596 \mathrm{E}+10$ \\
\hline MODAL & Mode & 4.000000 & 4662.3598 & 0.00000 & 0.00000 & 0.00000 & 641771729 & -340378541 \\
\hline MODAL & Mode & 5.000000 & 6322.5890 & 0.00000 & 0.00000 & 0.00000 & 9.00000 & 6.00000 \\
\hline MODAL & Mode & 6.000000 & -9145.8816 & 0.00000 & 0.00000 & 0.00000 & 9.00000 & 6.0000 \\
\hline MODAL & Mode & 7.000000 & 8376.2451 & 0.00000 & 0.00000 & 0.00000 & -39462048 & 13979686.08 \\
\hline MODAL & Mode & 8.000000 & -156.4183 & 0.00000 & 0.00000 & 0.00000 & -2468.97959 & -23755.4285 \\
\hline MODAL & Mode & 9.000000 & 409.1744 & 0.00000 & 0.00000 & 0.00000 & 9.00000 & 6.00000 \\
\hline MODAL & Mode & 10.000000 & 808.5365 & 0.00000 & 0.00000 & 0.00000 & 4037555374 & 433493416 . \\
\hline MODAL & Mode & 11.000000 & 3564.9829 & 0.00000 & 0.00000 & 0.00000 & 9.00000 & 6.0000 \\
\hline MODAL & Mode & 12.000000 & 2396.1176 & 0.00000 & 0.00000 & 0.00000 & 9.00000 & 6.00000 \\
\hline RS & $\operatorname{Max}$ & & 2846.9972 & 0.00000 & 0.00000 & 0.00000 & 17.62236 & $12.0418:$ \\
\hline
\end{tabular}

Table: Base Reactions, Part 3 of 4

$\begin{array}{rrrrrrrrr}\text { DEAD } & & & 0.00000 & -4.609 \mathrm{E}+10 & 1.599 \mathrm{E}+11 & 0.00000 & 9.00000 & 6.00000 \\ \text { MODAL } & \text { Mode } & 1.000000 & 0.00000 & 9.00000 & 6.00000 & 0.00000 & -317218067 & 1357682.622 \\ \text { MODAL } & \text { Mode } & 2.000000 & 0.00000 & 9.00000 & 6.00000 & 0.00000 & 2256909.783 & -248283100 \\ \text { MODAL } & \text { Mode } & 3.000000 & 0.00000 & -7256407324 & 350741759 . & 0.00000 & 9.00023 & 6.00097 \\ \text { MODAL } & \text { Mode } & 4.000000 & 0.00000 & -230924252 & -155268135 & 0.00000 & 9.00000 & 6.00000 \\ \text { MODAL } & \text { Mode } & 5.000000 & 0.00000 & 9.00001 & 6.00000 & 0.00000 & -6344765.9 & 34881164.2 \\ \text { MODAL } & \text { Mode } & 6.000000 & 0.00000 & 9.00000 & 6.00000 & 0.00000 & -108759225 & 125030128.9 \\ \text { MODAL } & \text { Mode } & 7.000000 & 0.00000 & -385419823 & -301111963 & 0.00000 & 9.00001 & 6.00002 \\ \text { MODAL } & \text { Mode } & 8.000000 & 0.00000 & 15352.79756 & -2723.96209 & 0.00000 & 9.00000 & 5.99999 \\ \text { MODAL } & \text { Mode } & 9.000000 & 0.00000 & 9.00001 & 6.00001 & 0.00000 & 6.075 \mathrm{E}+10 & -5815554671 \\ \text { MODAL } & \text { Mode } & 10.000000 & 0.00000 & 1201896.381 & -3279428.7 & 0.00000 & 9.00000 & 5.99999 \\ \text { MODAL } & \text { Mode } & 11.000000 & 0.00000 & 8.99999 & 6.00000 & 0.00000 & 1977237.129 & -348945.76 \\ \text { MODAL } & \text { Mode } & 12.000000 & 0.00000 & 8.99999 & 5.99999 & 0.00000 & 73377.41477 & -270105.498 \\ \text { RS } & \text { Max } & & 0.00000 & 17.70799 & 11.70814 & 0.00000 & 608763910 . & 5639918.05\end{array}$

Table: Base Reactions, Part 4 of 4

OutputCase StepType StepNum ZCentroidEZ

$\begin{array}{llll}\text { DEAD } & & & 0.00000 \\ \text { MODAL } & \text { Mode } & 1.000000 & 0.00000 \\ \text { MODAL } & \text { Mode } & 2.000000 & 0.00000 \\ \text { MODAL } & \text { Mode } & 3.000000 & 0.00000 \\ \text { MODAL } & \text { Mode } & 4.000000 & 0.00000\end{array}$




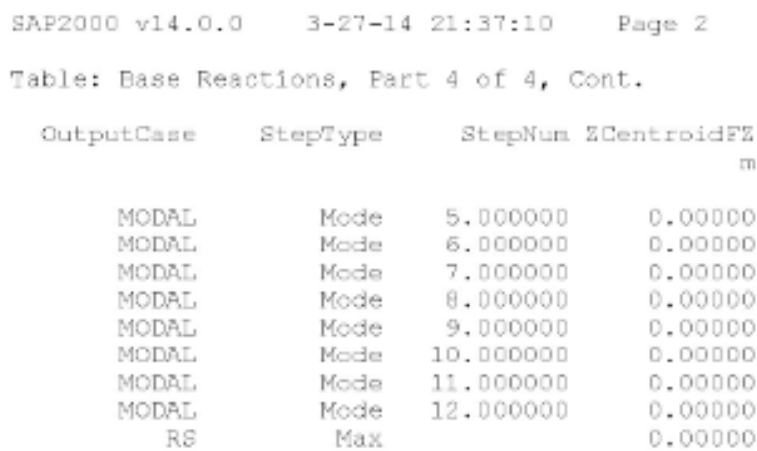

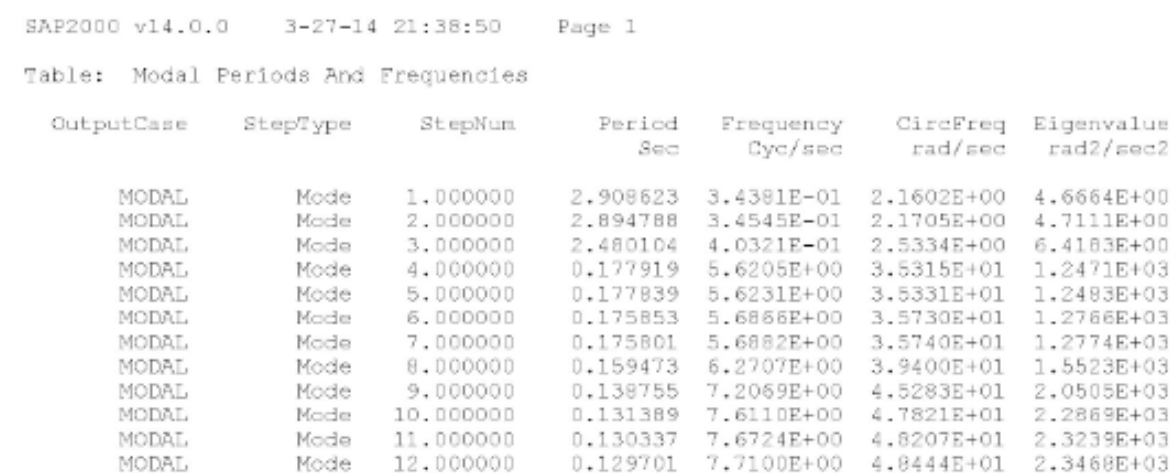

BASE REACTIONS

\begin{tabular}{|l|l|l|}
\hline \multicolumn{4}{|c|}{ RS Lin Resp. Spec.(max) } & GLOBAL MZ (KN-m) \\
\hline GLOBAL FX (KN) & GLOBAL MY (KN-m) & 2846.99 \\
\hline 474.46 & 972.643 & \\
\hline
\end{tabular}

RESULTS OF MODEL 8: 


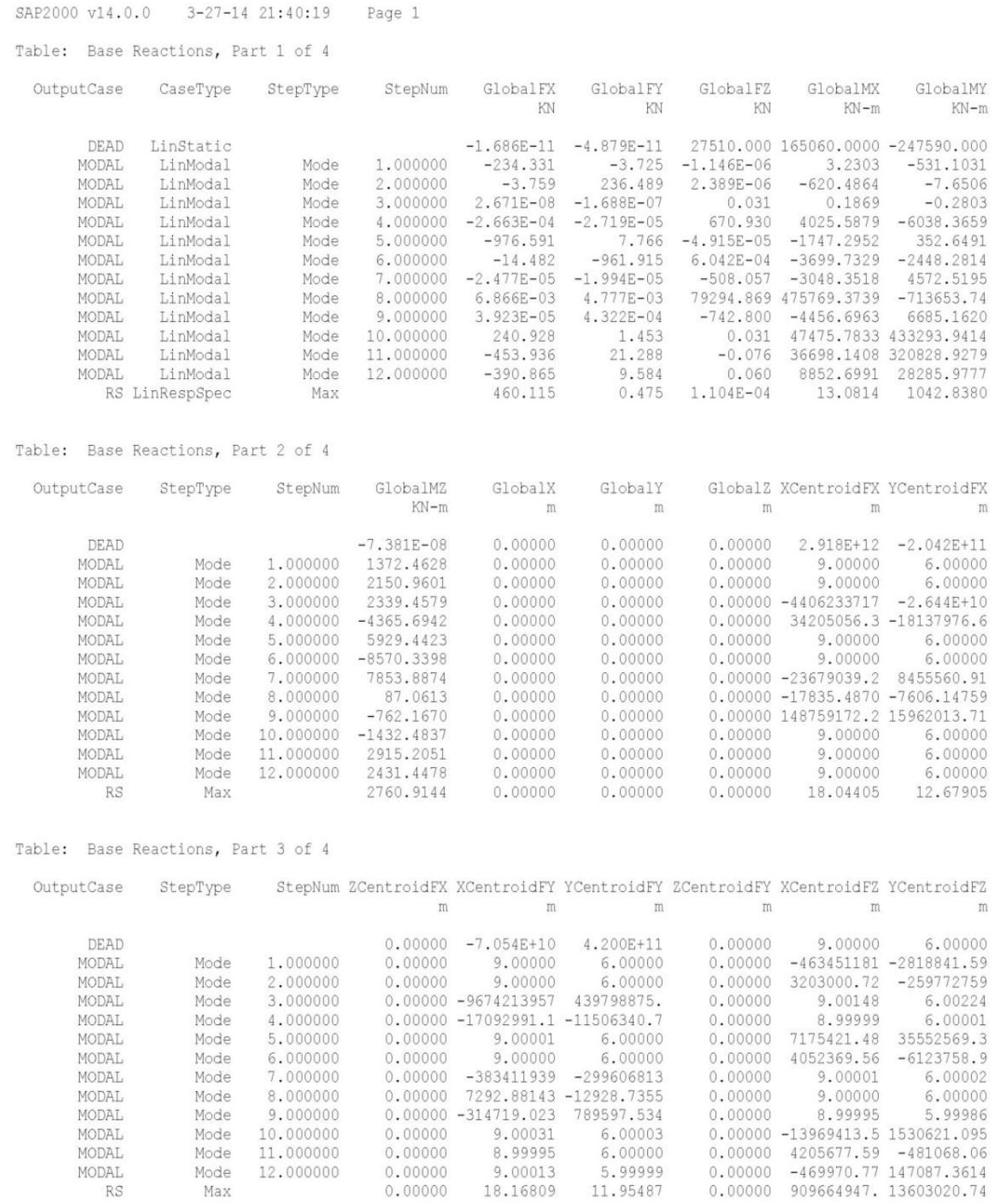

Table: Base Reactions, Part 4 of 4

$\begin{array}{rrrr}\text { OutputCase } & \text { StepType } & \text { StepNum } & \text { ZCentroidEZ } \\ & & & \\ \text { DEAD } & & & 0.00000 \\ \text { MODAL } & \text { Mode } & 1.000000 & 0.00000 \\ \text { MODAL } & \text { Mode } & 2.000000 & 0.00000 \\ \text { MODAL } & \text { Mode } & 3.000000 & 0.00000 \\ \text { MODAL } & \text { Mode } & 4.000000 & 0.00000\end{array}$




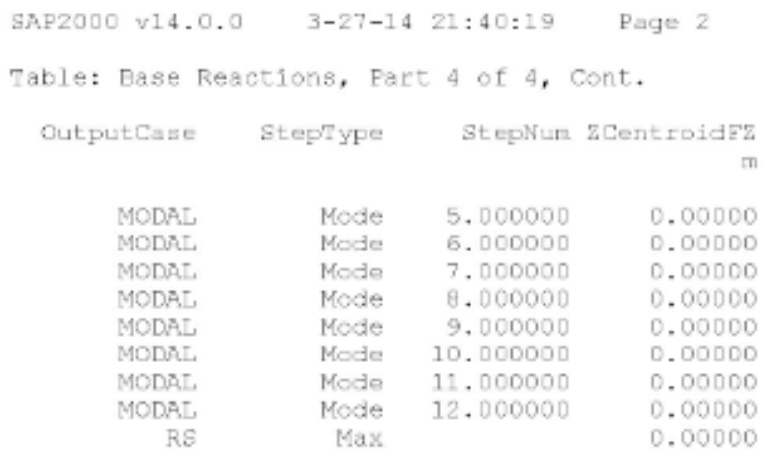

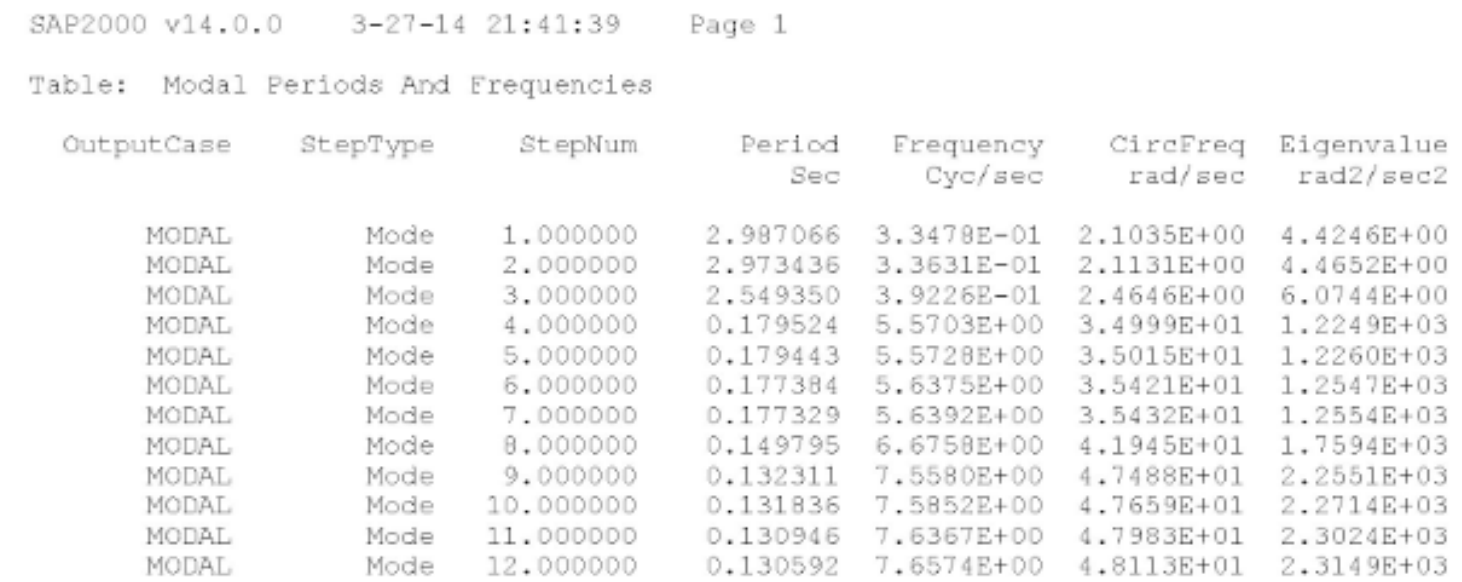

BASE REACTIONS

\begin{tabular}{|l|l|l|}
\hline \multicolumn{4}{|c|}{ RS Lin Resp. Spec. (max) } \\
\hline GLOBAL FX (KN) & GLOBAL MY (KN-m) & GLOBAL MZ (KN-m) \\
\hline 460.115 & 1042.84 & 2760.914 \\
\hline
\end{tabular}

RESULTS OF MODEL 9: 
SAP2000 v14.0.0 3-27-14 21:44:47

Table: Base Reactions, Part 1 of 4

$\begin{array}{rrr}\text { OutputCase } & \text { CaseType } & \text { StepType } \\ \text { DEAD } & \text { LinStatic } & \\ \text { MODAL } & \text { LinModal } & \text { Mode } \\ \text { MODAL } & \text { LinModal } & \text { Mode } \\ \text { MODAL } & \text { LinModal } & \text { Mode } \\ \text { MODAL } & \text { LinModal } & \text { Mode } \\ \text { MODAL } & \text { LinModal } & \text { Mode } \\ \text { MODAL } & \text { LinModal } & \text { Mode } \\ \text { MODAL } & \text { LinModal } & \text { Mode } \\ \text { MODAL } & \text { LinModal } & \text { Mode } \\ \text { MODAL } & \text { LinModal } & \text { Mode } \\ \text { MODAL } & \text { LinModal } & \text { Mode } \\ \text { MODAL } & \text { LinModal } & \text { Mode } \\ \text { MODAL } & \text { LinModal } & \text { Mode } \\ \text { RS } & \text { LinRespSpec } & \text { Max }\end{array}$

RS LinRespSpec Max
Page 1

Table: Base Reactions, Part 2 of 4

$\begin{array}{rrrr}\text { OutputCase } & \text { StepType } & \text { StepNum } & \begin{array}{r}\text { GlobalMZ } \\ \text { KN-m }\end{array} \\ \text { DEAD } & & & -1.637 \mathrm{E}-11 \\ \text { MODAL } & \text { Mode } & 1.000000 & -35263.2495 \\ \text { MODAL } & \text { Mode } & 2.000000 & -325286.67 \\ \text { MODAL } & \text { Mode } & 3.000000 & -0.0044 \\ \text { MODAL } & \text { Mode } & 4.000000 & -612871.19 \\ \text { MODAL } & \text { Mode } & 5.000000 & 1565.1887 \\ \text { MODAL } & \text { Mode } & 6.000000 & -8497.8607 \\ \text { MODAL } & \text { Mode } & 7.000000 & 0.0952 \\ \text { MODAL } & \text { Mode } & 8.000000 & -1844.4034 \\ \text { MODAL } & \text { Mode } & 9.000000 & -152.2371 \\ \text { MODAL } & \text { Mode } & 10.000000 & 0.1851 \\ \text { MODAL } & \text { Mode } & 11.000000 & -0.0140 \\ \text { MODAL } & \text { Mode } & 12.000000 & -2617.1729 \\ \text { RS } & \text { Max } & & 16684.9908\end{array}$

$\begin{array}{rr}\text { StepNum } & \begin{array}{r}\text { Global EX } \\ \mathrm{KN}\end{array} \\ & 1.705 \mathrm{E}-12 \\ 1.000000 & -1.094 \mathrm{E}-07 \\ 2.000000 & -2.991 \mathrm{E}-04 \\ 3.000000 & 7.842 \mathrm{E}-04 \\ 4.000000 & 102145.197 \\ 5.000000 & -1.439 \mathrm{E}-03 \\ 6.000000 & 1416.314 \\ 7.000000 & -5.645 \mathrm{E}-03 \\ 8.000000 & 0.011 \\ 9.000000 & 0.015 \\ 10.000000 & -0.037 \\ 11.000000 & 1.211 \mathrm{E}-03 \\ 12.000000 & -6.502 \mathrm{E}-03 \\ & \end{array}$

GlobalFY

GlobalE

GlobalMX

GlobalMY $\begin{array}{llll}-3918.139 & -3.578 \mathrm{E}-06 & 14078.2281 & 2.826 \mathrm{E}-05\end{array}$ $\begin{array}{rrrr}5.935 \mathrm{E}-06 & 1.011 \mathrm{E}-03 & 0.0568 & -0.0161\end{array}$ $\begin{array}{llll}-2.649 \mathrm{E}-05 & 78921.849 & 473531.0700 & -710296.71\end{array}$ $\begin{array}{lll}-1.811 \mathrm{E}-04 & 1.343 \mathrm{E}-03 & 0.0147 \quad 345055.9376\end{array}$ $\begin{array}{llll}173.910 & 0.032 & 373413.1177 & -0.0152\end{array}$ $\begin{array}{llll}-9.266 \mathrm{E}-04 & -7.509 \mathrm{E}-03 & -0.6350 & -585076.26\end{array}$

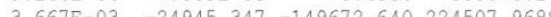
$\begin{array}{rrrr}-.667 \mathrm{E}-03 & -24945.347 & -149672.640 & 224507.9688 \\ -6.314 \mathrm{E}-03 & -0.069 & -1.1291 & -0.5970\end{array}$ $\begin{array}{rrrr}-6.314 \mathrm{E}-03 & -0.069 & -1.1291 & -0.5970 \\ -16.916 & 0.069 & -100132.477 & -1.6963\end{array}$ $\begin{array}{llll}3.374 \mathrm{E}-03 & 35805.638 & 214834.9514 & -322248.44\end{array}$

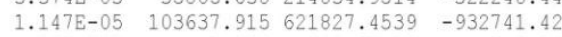
$\begin{array}{llll}-290.802 & 0.020 & -344455.58 & 0.0438\end{array}$ $2780.832 \quad 4.954 \mathrm{E}-06$

$\begin{array}{rr}\begin{array}{rr}\text { GlobalX } \\ \text { m }\end{array} & \text { GlobalY } \\ & \text { m } \\ 0.00000 & 0.00000 \\ 0.00000 & 0.00000 \\ 0.00000 & 0.00000 \\ 0.00000 & 0.00000 \\ 0.00000 & 0.00000 \\ 0.00000 & 0.00000 \\ 0.00000 & 0.00000 \\ 0.00000 & 0.00000 \\ 0.00000 & 0.00000 \\ 0.00000 & 0.00000 \\ 0.00000 & 0.00000 \\ 0.00000 & 0.00000 \\ 0.00000 & 0.00000 \\ 0.00000 & 0.00000\end{array}$

Globalz XCentroidEX YCentroidEX

$0.00000 \quad-1.028 \mathrm{E}+16 \quad 8.00000$ $0.00000 \quad-17.52084 \quad 14.50371$ $0.00000-33.77804-1003358212$

$0.00000-66651388 . \quad 5.46756$

$0.00000 \quad 9.00000 \quad 6.00000$

$\begin{array}{lll}0.00000 & 45.33056 \quad 0.39802\end{array}$

$\begin{array}{lll}0.00000 & 9.00004 & 5.99998\end{array}$

$0.00000 \quad 6663902.88 \quad 11.18595$

$0.00000 \quad 25.14700 \quad 255479.9693$

$\begin{array}{lll}0.00000 & 26.31309 & -0.97129\end{array}$

$0.00000 \quad 519765.516 \quad 4.14894$

$0.00000-52021844 . \quad 11.41165$

$\begin{array}{lll}0.00000 & 8.80095 & 6.50902\end{array}$

$\begin{array}{lll}0.00000 & 0.24329 & 0.16760\end{array}$

Table: Base Reactions, Part 3 of 4

\begin{tabular}{|c|c|c|c|c|c|c|c|c|}
\hline OutputCase & StepType & StepNum & ZCentroidEX & XCentroidFY & YCentroidFY & ZCentroidFY & XCentroidEZ & YCentroidEZ \\
\hline & & & $\mathrm{m}$ & $\mathrm{m}$ & $\mathrm{m}$ & $\mathrm{m}$ & $\mathrm{m}$ & \\
\hline DEAD & & & 0.00000 & -44.15898 & $-4.119 E+15$ & 0.00000 & 9.00000 & 6.00000 \\
\hline MODAL & Mode & 1.000000 & 0.00000 & 9.00000 & 6.00000 & 0.00000 & 7.84219 & -1301774266 \\
\hline MODAL & Mode & 2.000000 & 0.00000 & -4133231539 & 1154.40350 & 0.00000 & 15.99104 & 56.21763 \\
\hline MODAL & Mode & 3.000000 & 0.00000 & 2.63510 & 9191841.28 & 0.00000 & 9.00000 & 6.00000 \\
\hline MODAL & Mode & 4.000000 & 0.00000 & 9.22162 & 8.80485 & 0.00000 & -249095294 & 10.76204 \\
\hline MODAL & Mode & 5.000000 & 0.00000 & 9.00000 & 5.99998 & 0.00000 & 0.41516 & 11745671.41 \\
\hline MODAL & Mode & 6.000000 & 0.00000 & 9.03701 & 16.08351 & 0.00000 & -77942357. & 84.69883 \\
\hline MODAL & Mode & 7.000000 & 0.00000 & 8.74994 & 43394.70434 & 0.00000 & 8.99999 & 6.00002 \\
\hline MODAL & Mode & 8.000000 & 0.00000 & -157045.692 & 5.87323 & 0.00000 & -8.71877 & 16.49702 \\
\hline MODAL & Mode & 9.000000 & 0.00000 & 9.00049 & 5.99820 & 0.00000 & 24.53951 & -1447706.27 \\
\hline MODAL & Mode & 10.000000 & 0.00000 & 8.97381 & 516094.619 & 0.00000 & 8.99994 & 6.00003 \\
\hline MODAL & Mode & 11.000000 & 0.00000 & -16.40236 & -129941046 & 0.00000 & 9.00000 & 6.00000 \\
\hline MODAL & Mode & 12.000000 & 0.00000 & 9.00000 & 6.00000 & 0.00000 & -2.01285 & -17679501.7 \\
\hline RS & $\operatorname{Max}$ & & 0.00000 & 0.28320 & 0.24048 & 0.00000 & 6782933.62 & 0.29468 \\
\hline
\end{tabular}

Table: Base Reactions, Part 4 of 4

$\begin{array}{rrrr}\text { OutputCase } & \text { StepType } & \text { StepNum } & \text { ZCentroidFZ } \\ & & & \\ \text { DEAD } & & & 0.00000 \\ \text { MODAL } & \text { Mode } & 1.000000 & 0.00000 \\ \text { MODAL } & \text { Mode } & 2.000000 & 0.00000 \\ \text { MODAL } & \text { Mode } & 3.000000 & 0.00000 \\ \text { MODAL } & \text { Mode } & 4.000000 & 0.00000\end{array}$




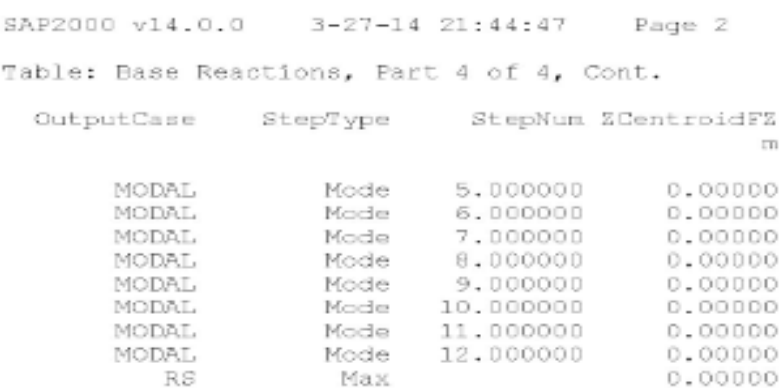

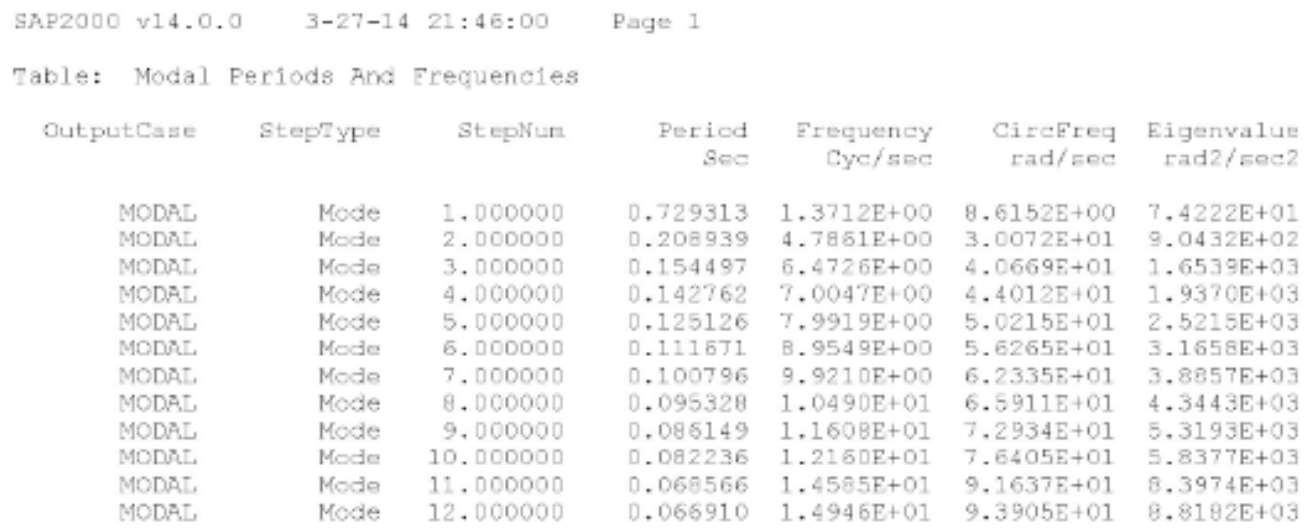

BASE REACTIONS

\begin{tabular}{|l|l|l|}
\hline \multicolumn{4}{|c|}{ RS Lin Resp. Spec. (max) } & GLOBAL MZ (KN-m) \\
\hline GLOBAL FX (KN) & GLOBAL MY (KN-m) & 16684.99 \\
\hline 2780.832 & 9382.55 &
\end{tabular}

\section{RESULTS OF MODEL 10:}


SAP2000 v14.0.0 3-27-14 21:47:28

Table: Base Reactions, Part 1 of 4

$\begin{array}{ccc}\text { OutputCase } & \text { CaseType } & \text { StepType } \\ & & \\ \text { DEAD } & \text { LinStatic } & \\ \text { MODAL } & \text { LinModal } & \text { Mode } \\ \text { MODAL } & \text { LinModal } & \text { Mode } \\ \text { MODAL } & \text { LinModal } & \text { Mode } \\ \text { MODAL } & \text { LinModal } & \text { Mode } \\ \text { MODAL } & \text { LinModal } & \text { Mode } \\ \text { MODAL } & \text { LinModal } & \text { Mode } \\ \text { MODAL } & \text { LinModal } & \text { Mode } \\ \text { MODAL } & \text { LinModal } & \text { Mode } \\ \text { MODAL } & \text { LinModal } & \text { Mode } \\ \text { MODAL } & \text { LinModal } & \text { Mode } \\ \text { MODAL } & \text { LinModal } & \text { Mode } \\ \text { MODAL } & \text { LinModal } & \text { Mode } \\ \text { RS } & \end{array}$

$\begin{array}{rr}\text { StepNum } & \begin{array}{r}\text { Global EX } \\ \mathrm{KN}\end{array} \\ & 3.801 \mathrm{E}-13 \\ & 3.759 \mathrm{E}-08 \\ 1.000000 & 4.188 \mathrm{E}-05 \\ 2.000000 & 55654.556 \\ 3.000000 & 5 \\ 4.000000 & -1.597 \mathrm{E}-04 \\ 5.000000 & -5.383 \mathrm{E}-05 \\ 6.000000 & 224.558 \\ 7.000000 & 2.981 \mathrm{E}-04 \\ 8.000000 & 2.783 \mathrm{E}-04 \\ 9.000000 & -5.897 \mathrm{E}-03 \\ 10.000000 & -7.065 \mathrm{E}-04 \\ 11.000000 & -4.536 \mathrm{E}-04 \\ 12.000000 & 1.231 \mathrm{E}-03 \\ & 2438.178\end{array}$

GlobalFY

$\mathrm{KN}$

$-2.398 \mathrm{E}-14$ 3985.586
$5.5395-05$
$6.8535-05$

$6.853 \mathrm{E}-05$

$1.835 \mathrm{E}-03$

$1.835 \mathrm{E}-03$
-200.862

$2.225 \mathrm{E}-03$

$-0.013$

$3.742 \mathrm{E}-03$

-3.337
$532 \mathrm{E}-03$

$5.532 \mathrm{E}-03$

$-57.896$

3. $004 \mathrm{E}-06$

GlobalEZ

GlobalMX

GlobalmY

$26880.427161282 .5633-241923.845$ $-9.574 \mathrm{E}-06-14366.9800 \quad 6.165 \mathrm{E}-05$ $\begin{array}{lll}-1.706 \mathrm{E}-03 & -0.0067 & 0.0161\end{array}$ $8.962 \mathrm{E}-04 \quad-0.0017246114 .4220$ $69795.586418773 .3752-628160.23$ $0.027-334075.66 \quad 0.0069$ $0.031 \quad-0.1131 \quad-581585.20$ $31326.927 \quad 187963.4288-281943.117$ $\begin{array}{lll}-0.086 & -1.1949 & 0.4174\end{array}$ $-0.257 \quad 126107.8668 \quad 1.3725$ $33183.064199099 .3576-298648.805$ $3346.08120076 .1949-30114.7487$ $\begin{array}{lll}0.027 & -3859.2622 & -0.6658\end{array}$ $\begin{array}{lll}3.903 \mathrm{E}-05 & 7.833 \mathrm{E}-05 & 10781.6702\end{array}$

Table: Base Reactions, Part 2 of 4

\begin{tabular}{|c|c|c|c|c|c|c|c|c|}
\hline OutputCase & StepType & StepNum & $\begin{array}{r}\text { GlobalMZ } \\
\mathrm{KN}-\mathrm{m}\end{array}$ & $\begin{array}{r}\text { GlobalX } \\
\mathrm{m}\end{array}$ & $\begin{array}{r}\text { GlobalY } \\
\mathrm{m}\end{array}$ & $\begin{array}{r}\text { Globalz } \\
\mathrm{m}\end{array}$ & $\begin{array}{r}\text { XCentroidEX } \\
\mathrm{m}\end{array}$ & $\begin{array}{r}\text { YCentroidEX } \\
\mathrm{m}\end{array}$ \\
\hline DEAD & & & $-6.310 \mathrm{E}-12$ & 0.00000 & 0.00000 & 0.00000 & $-2.791 E+15$ & 10.46729 \\
\hline MODAL & Mode & 1.000000 & 35870.2704 & 0.00000 & 0.00000 & 0.00000 & 85.96911 & -3.83566 \\
\hline MODAL & Mode & 2.000000 & -187281.603 & 0.00000 & 0.00000 & 0.00000 & 25.25802 & 3872522735 \\
\hline MODAL & Mode & 3.000000 & -333927.33 & 0.00000 & 0.00000 & 0.00000 & 9.00000 & 6.00000 \\
\hline MODAL & Mode & 4.000000 & 0.0164 & 0.00000 & 0.00000 & 0.00000 & 10849509.56 & -0.63559 \\
\hline MODAL & Mode & 5.000000 & -1807.7560 & 0.00000 & 0.00000 & 0.00000 & 88.33090 & 76.12884 \\
\hline MODAL & Mode & 6.000000 & -1347.3346 & 0.00000 & 0.00000 & 0.00000 & 8.99998 & 6.00002 \\
\hline MODAL & Mode & 7.000000 & -0.1081 & 0.00000 & 0.00000 & 0.00000 & 6214933.86 & -36.69890 \\
\hline MODAL & Mode & 8.000000 & -667.6535 & 0.00000 & 0.00000 & 0.00000 & 127.44467 & -1194219.60 \\
\hline MODAL & Mode & 9.000000 & -30.0049 & 0.00000 & 0.00000 & 0.00000 & -15.11273 & 4.84733 \\
\hline MODAL & Mode & 10.000000 & 0.0636 & 0.00000 & 0.00000 & 0.00000 & 729405.053 & 16.47158 \\
\hline MODAL & Mode & 11.000000 & -0.0028 & 0.00000 & 0.00000 & 0.00000 & 996778.590 & -2.44677 \\
\hline MODAL & Mode & 12.000000 & -521.0733 & 0.00000 & 0.00000 & 0.00000 & 10.17329 & 8.74332 \\
\hline RS & Max & & 14629.0704 & 0.00000 & 0.00000 & 0.00000 & 0.39424 & 0.26551 \\
\hline
\end{tabular}

Table: Base Reactions, Part 3 of 4

\begin{tabular}{|c|c|c|c|c|c|c|c|c|}
\hline OutputCase & StepType & St epNum & ZCentroidEX & XCentroidFY & YCentroidEY & ZCentroidEY & XCentroidEZ & YCentroidEZ \\
\hline & & & $\mathrm{m}$ & $\mathrm{m}$ & $\mathrm{m}$ & m & $\mathrm{m}$ & $\mathrm{m}$ \\
\hline DEAD & & & 0.00000 & 92.81481 & $6.147 \mathrm{E}+15$ & 0.00000 & 9.00000 & 6.00000 \\
\hline MODAL & Mode & 1.000000 & 0.00000 & 9.00000 & 6.00000 & 0.00000 & 6.43425 & 480482150 . \\
\hline MODAL & Mode & 2.000000 & 0.00000 & -439689873 & 6.06545 & 0.00000 & 9.41359 & 3.81210 \\
\hline MODAL & Mode & 3.000000 & 0.00000 & 9.21735 & 14.77402 & 0.00000 & -261239425 & -1.69895 \\
\hline MODAL & Mode & 4.000000 & 0.00000 & 9.01256 & -126743.585 & 0.00000 & 9.00000 & 6.00000 \\
\hline MODAL & Mode & 5.000000 & 0.00000 & 9.00000 & 6.00008 & 0.00000 & -0.25956 & -12440725.8 \\
\hline MODAL & Mode & 6.000000 & 0.00000 & 8.80363 & 3.07368 & 0.00000 & 18591890.09 & -3.31143 \\
\hline MODAL & Mode & 7.000000 & 0.00000 & 9.08786 & 12173.38455 & 0.00000 & 9.00002 & 6.00006 \\
\hline MODAL & Mode & 8.000000 & 0.00000 & -267308.876 & 12.02353 & 0.00000 & 4.84287 & 13.75104 \\
\hline MODAL & Mode & 9.000000 & 0.00000 & 9.00016 & 5.98978 & 0.00000 & 5.37410 & -490535.14 \\
\hline MODAL & Mode & 10.000000 & 0.00000 & 9.39926 & 314891.3964 & 0.00000 & 9.00004 & 6.00003 \\
\hline MODAL & Mode & 11.000000 & 0.00000 & 8.75840 & -44236951 & 0.00000 & 9.00001 & 5.99991 \\
\hline MODAL & Mode & 12.000000 & 0.00000 & 8.99999 & 6.00001 & 0.00000 & 24.73238 & -156504.547 \\
\hline RS & $\operatorname{Max}$ & & 0.00000 & 0.40363 & 0.64726 & 0.00000 & 11444661.96 & 0.07442 \\
\hline
\end{tabular}

Table: Base Reactions, Part 4 of 4

$\begin{array}{rrrr}\text { OutputCase } & \text { StepType } & \text { StepNum ZCentroidEZ } \\ & & & \\ \text { DEAD } & & & 0.00000 \\ \text { MODAL } & \text { Mode } & 1.000000 & 0.00000 \\ \text { MODAL } & \text { Mode } & 2.000000 & 0.00000 \\ \text { MODAL } & \text { Mode } & 3.000000 & 0.00000 \\ \text { MODAL } & \text { Mode } & 4.000000 & 0.00000\end{array}$




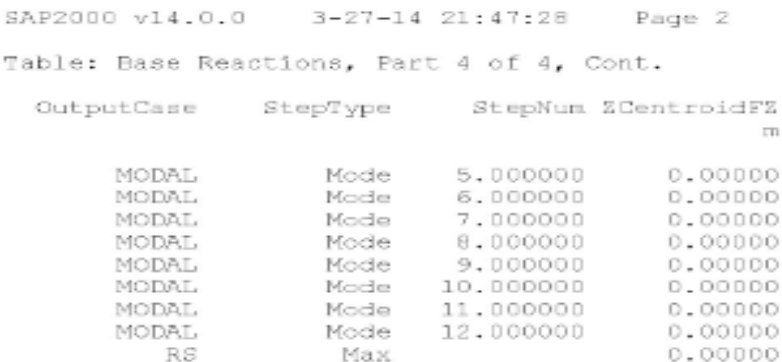

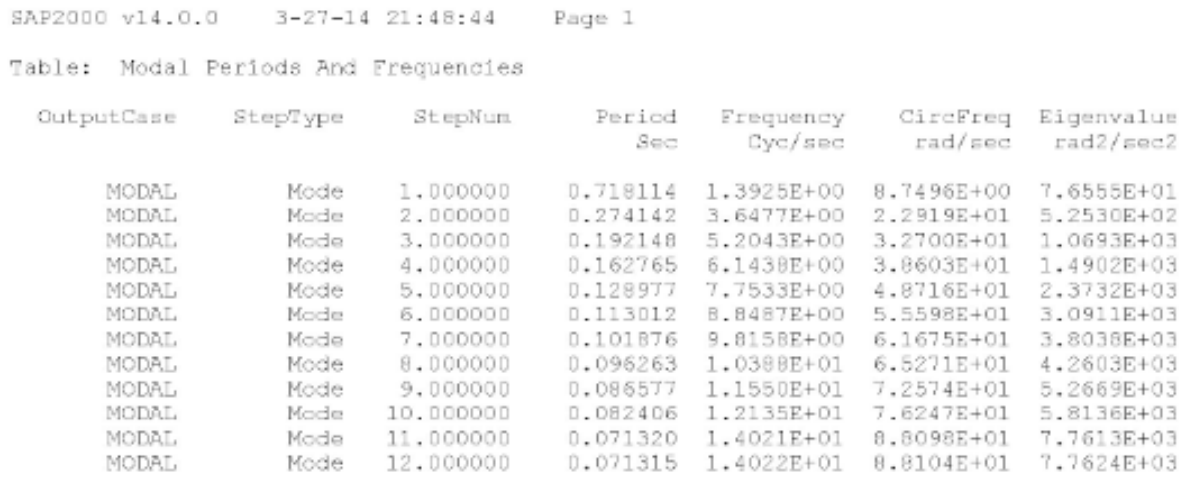

BASE REACTIONS

RS Lin Resp. Spec.(max)

\begin{tabular}{|l|l|l|l|}
\hline \multicolumn{4}{|c|}{ RS Lin Resp. Spec.(max) } \\
\hline GLOBAL FX (KN) & GLOBAL MY (KN-m) & GLOBAL MZ (KN-m) \\
\hline 2438.178 & 10781.67 & 14629 \\
\hline
\end{tabular}

RESULTS OF MODEL 1: 


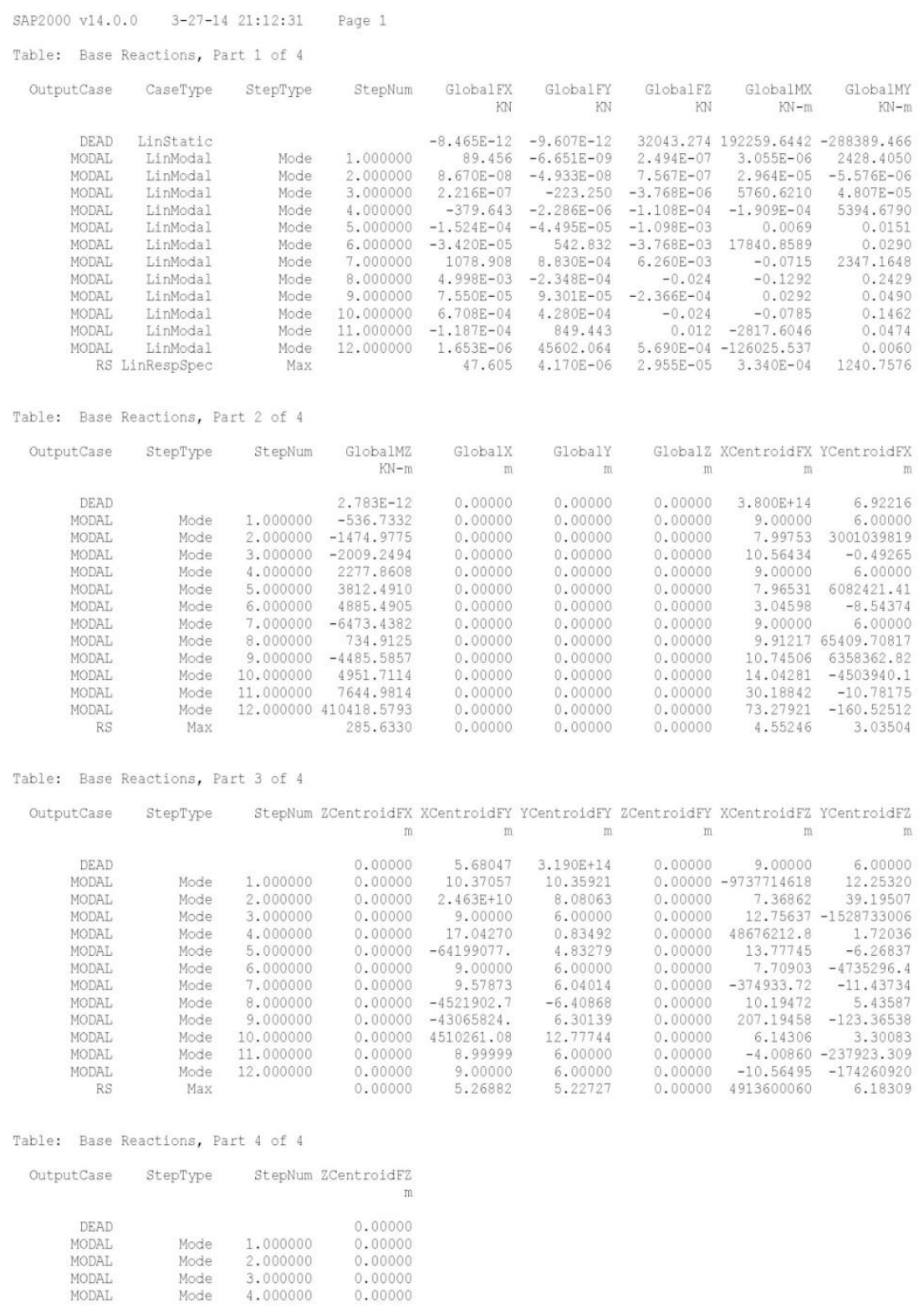




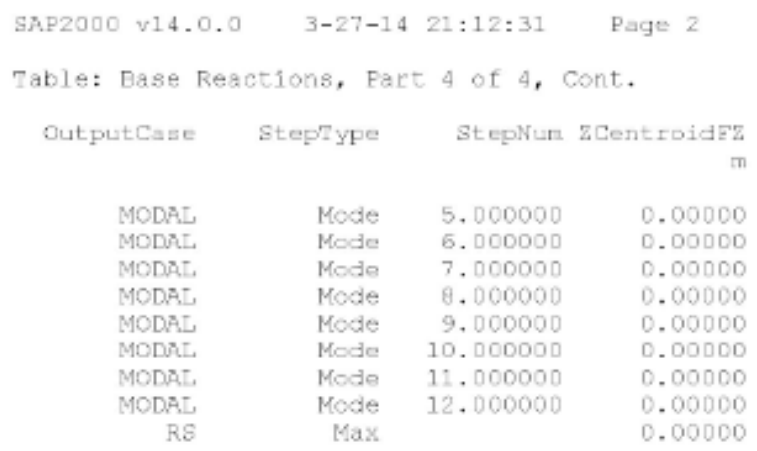

SAP2000 v14.0.0 3-27-14 21:16:22 Page 1

Table: Modal Periods And Erequencies

$\begin{array}{lccrrrr}\text { OutputCase } & \text { StepType } & \text { StepNum } & \begin{array}{r}\text { Period } \\ \text { Sec }\end{array} & \begin{array}{r}\text { Frequency } \\ \text { Cyc/sec }\end{array} & \begin{array}{r}\text { CircEreq } \\ \text { rad/sec }\end{array} & \begin{array}{r}\text { Eigenvalue } \\ \text { rad2/sec2 }\end{array} \\ \text { MODAL } & \text { Mode } & 1.000000 & 2.613525 & 3.8263 \mathrm{E}-01 & 2.4041 \mathrm{E}+00 & 5.7797 \mathrm{E}+00 \\ \text { MODAL } & \text { Mode } & 2.000000 & 1.772198 & 5.6427 \mathrm{E}-01 & 3.5454 \mathrm{E}+00 & 1.2570 \mathrm{E}+01 \\ \text { MODAL } & \text { Mode } & 3.000000 & 1.680376 & 5.9510 \mathrm{E}-01 & 3.7392 \mathrm{E}+00 & 1.3981 \mathrm{E}+01 \\ \text { MODAL } & \text { Mode } & 4.000000 & 0.667218 & 1.4988 \mathrm{E}+00 & 9.4170 \mathrm{E}+00 & 8.8680 \mathrm{E}+01 \\ \text { MODAL } & \text { Mode } & 5.000000 & 0.502527 & 1.9899 \mathrm{E}+00 & 1.2503 \mathrm{E}+01 & 1.5633 \mathrm{E}+02 \\ \text { MODAL } & \text { Mode } & 6.000000 & 0.478131 & 2.0915 \mathrm{E}+00 & 1.3141 \mathrm{E}+01 & 1.7269 \mathrm{E}+02 \\ \text { MODAL } & \text { Mode } & 7.000000 & 0.294187 & 3.3992 \mathrm{E}+00 & 2.1358 \mathrm{E}+01 & 4.5615 \mathrm{E}+02 \\ \text { MODAL } & \text { Mode } & 8.000000 & 0.282011 & 3.5460 \mathrm{E}+00 & 2.2280 \mathrm{E}+01 & 4.9639 \mathrm{E}+02 \\ \text { MODAL } & \text { Mode } & 9.000000 & 0.264587 & 3.7795 \mathrm{E}+00 & 2.3747 \mathrm{E}+01 & 5.6393 \mathrm{E}+02 \\ \text { MODAL } & \text { Mode } & 10.000000 & 0.247193 & 4.0454 \mathrm{E}+00 & 2.5418 \mathrm{E}+01 & 6.4608 \mathrm{E}+02 \\ \text { MODAL } & \text { Mode } & 11.000000 & 0.242641 & 4.1213 \mathrm{E}+00 & 2.5895 \mathrm{E}+01 & 6.7055 \mathrm{E}+02 \\ \text { MODAL } & \text { Mode } & 12.000000 & 0.216764 & 4.6133 \mathrm{E}+00 & 2.8986 \mathrm{E}+01 & 8.4021 \mathrm{E}+02\end{array}$

\begin{tabular}{|l|l|l|}
\hline \multicolumn{4}{|c|}{ RS Lin Resp. Spec. (max) } & GLOBAL MZ (KN-m) \\
\hline GLOBAL FX (KN) & GLOBAL MY (KN-m) & 285.63 \\
\hline 47.605 & 1240.75 & \\
\hline
\end{tabular}

\section{RESULTS OF MODEL 2:}




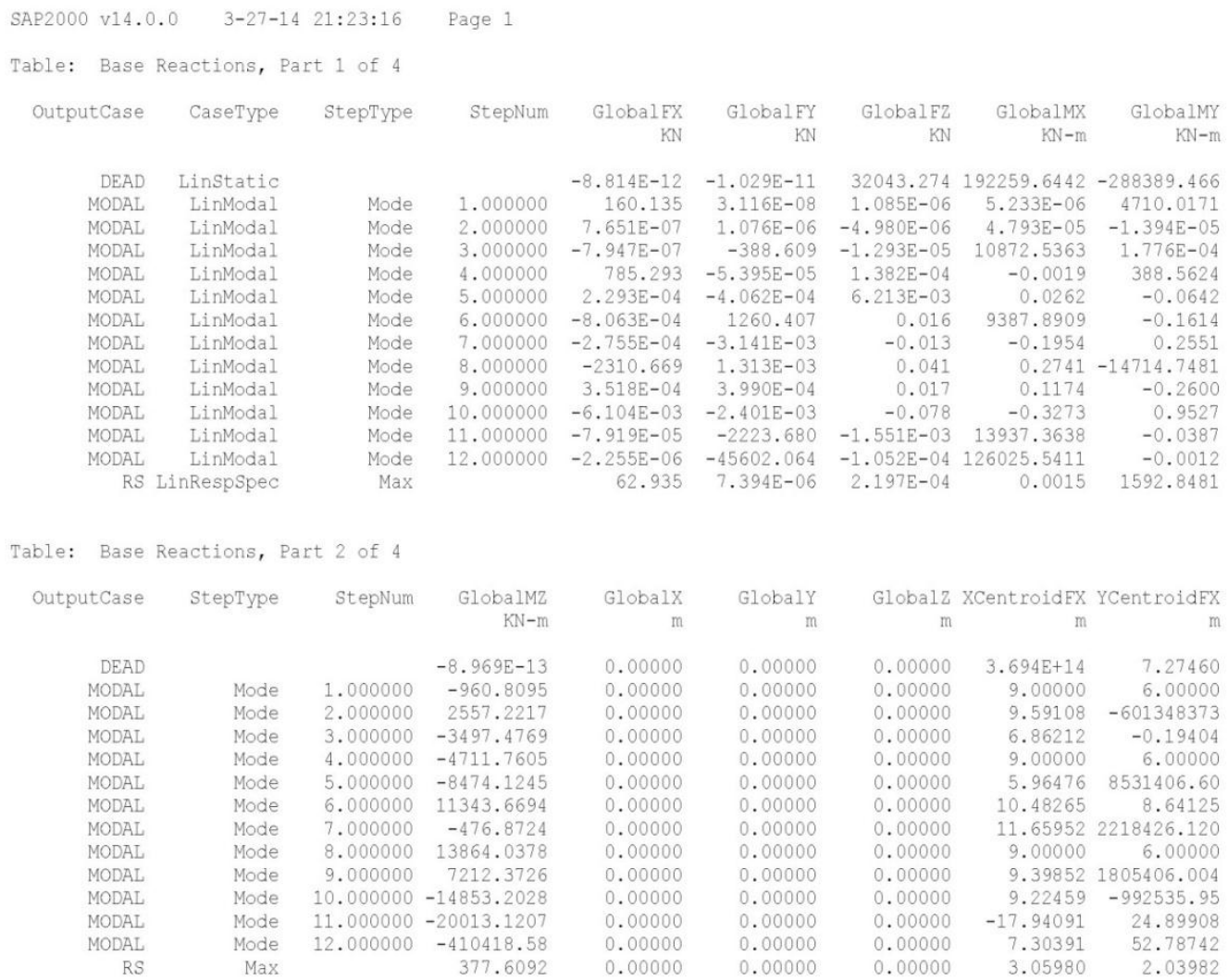

Table: Base Reactions, Part 3 of 4

\begin{tabular}{|c|c|c|c|c|c|c|c|c|}
\hline OutputCase & StepType & StepNum & $\begin{array}{r}\text { ZCentroidEX } \\
\mathrm{m}\end{array}$ & $\begin{array}{r}\text { XCentroidFY } \\
\mathrm{m}\end{array}$ & $\begin{array}{r}\text { YCentroidEY } \\
\mathrm{m}\end{array}$ & $\begin{array}{r}\text { ZCentroidEY } \\
\mathrm{m}\end{array}$ & XCentroidEZ & YCentroidEZ \\
\hline DEAD & & & 0.00000 & 6.27624 & $2.987 \mathrm{E}+14$ & 0.00000 & 9.00000 & 6.00000 \\
\hline MODAL & Mode & 1.000000 & 0.00000 & 10.23992 & 1.58397 & 0.00000 & -3067661688 & 4.90470 \\
\hline MODAL & Mode & 2.000000 & 0.00000 & 1941782821 & 6.44529 & 0.00000 & -3.27285 & -10.24511 \\
\hline MODAL & Mode & 3.000000 & 0.00000 & 9.00000 & 6.00000 & 0.00000 & 13.91490 & -714301750 \\
\hline MODAL & Mode & 4.000000 & 0.00000 & -0.82069 & 6.61680 & 0.00000 & 34189493.6 & -14.92955 \\
\hline MODAL & Mode & 5.000000 & 0.00000 & 15991494.77 & 7.25862 & 0.00000 & 10.44173 & 4.03908 \\
\hline MODAL & Mode & 6.000000 & 0.00000 & 9.00000 & 6.00000 & 0.00000 & 10.22649 & 927208.755 \\
\hline MODAL & Mode & 7.000000 & 0.00000 & 348939.589 & 5.83839 & 0.00000 & 20.00712 & 15.91413 \\
\hline MODAL & Mode & 8.000000 & 0.00000 & 9.53763 & 6.96914 & 0.00000 & 80921.97218 & 6.70154 \\
\hline MODAL & Mode & 9.000000 & 0.00000 & 19687520.45 & 7.69039 & 0.00000 & 15.01595 & 6.81170 \\
\hline MODAL & Mode & 10.000000 & 0.00000 & 3643900.45 & 6.80255 & 0.00000 & 12.48163 & 4.28481 \\
\hline MODAL & Mode & 11.000000 & 0.00000 & 9.00000 & 6.00000 & 0.00000 & -24.89712 & -3765840.6 \\
\hline MODAL & Mode & 12.000000 & 0.00000 & 9.00000 & 6.00000 & 0.00000 & -11.60662 & -942453623 \\
\hline RS & $\operatorname{Max}$ & & 0.00000 & 3.45882 & 0.59108 & 0.00000 & 1036026511 & 1.74572 \\
\hline
\end{tabular}

Table: Base Reactions, Part 4 of 4

$\begin{array}{rrrr}\text { OutputCase } & \text { StepType } & \text { StepNum ZCentroidEZ } \\ & & & \\ \text { DEAD } & & & 0.00000 \\ \text { MODAL } & \text { Mode } & 1.000000 & 0.00000 \\ \text { MODAL } & \text { Mode } & 2.000000 & 0.00000 \\ \text { MODAL } & \text { Mode } & 3.000000 & 0.00000 \\ \text { MODAL } & \text { Mode } & 4.000000 & 0.00000\end{array}$




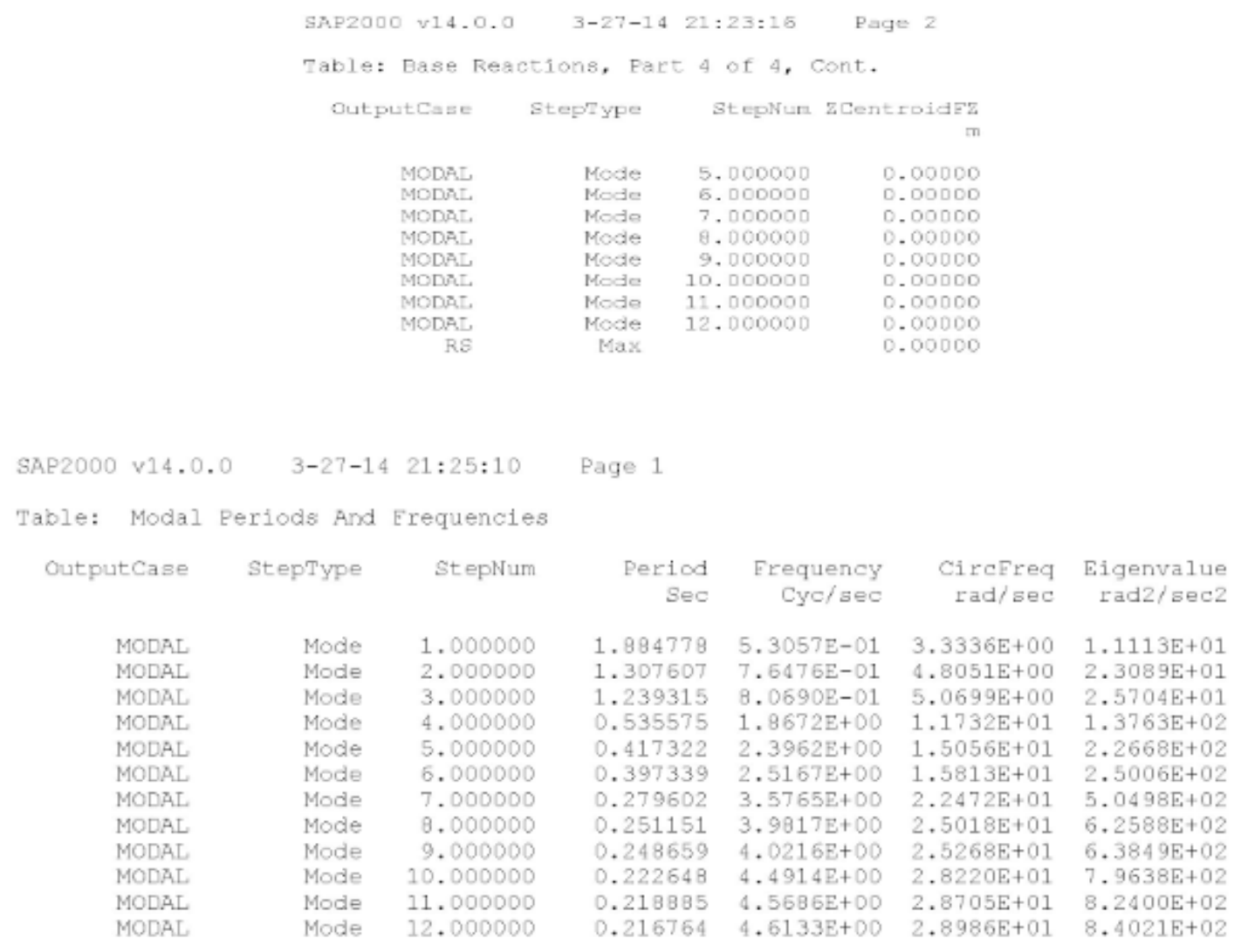

BASE REACTIONS

\begin{tabular}{|l|l|l|}
\hline \multicolumn{4}{|c|}{ BASE REACTIONS } \\
\hline \multicolumn{3}{|c|}{ RS Lin Resp. Spec. (max) } \\
\hline GLOBAL FX (KN) & GLOBAL MY (KN-m) & GLOBAL MZ (KN-m) \\
\hline 62.935 & 1592.8481 & 377.6092 \\
\hline
\end{tabular}

III. Final Conclusion:

1. From these results I have done a comparative study and I found that COMBINED CORE PILLAR CONCEPT is most effective.

Comparison between COMBINED CORE PILLAR CONCEPT and BASE ISOLATION(FRICTION ISOLATOR):

(A) The base reactions are found to be less as compared to base isolation.

(B) Base isolation systems are found useful for short period (Low Rise) structures, say less than 0.7 s including soil-structure interaction but Combined Core Pillar Concept can be used from Low to high rise building.

(C) In Torsional mode building performs well with Combined Core Pillar Concept than base isolation system used. 